\title{
Operator reflection positivity inequalities and their applications to interacting quantum rotors
}

\author{
Jacek Wojtkiewicz ${ }^{\dagger}$, Wiestaw Pusz ${ }^{\dagger}$, Piotr Stachura* \\ $\dagger$ Department for Mathematical Methods in Physics, \\ Faculty of Physics, Warsaw University, \\ Pasteura 5, 02-093 Warszawa, Poland \\ *The Faculty of Applied Informatics and Mathematics,
Warsaw University of Life Sciences-SGGW,
ul. Nowoursynowska 159, 02-776 Warszawa, Poland
}

October 15, 2018

\begin{abstract}
In the Reflection Positivity theory and its application to statistical mechanical systems, certain matrix inequalities play a central role. The Dyson-Lieb-Simon [1] and Kennedy-Lieb-Shastry [2] inequalities constitute prominent examples. In this paper we extend the KLS inequality to the case where matrices are replaced by certain operators. As an application, we prove the occurrence of the long range order in the ground state of two-dimensional quantum rotors.
\end{abstract}

Keywords: Statistical mechanics; phase transitions; operator inequalities; reflection positivity

\section{Introduction}

The Reflection Positivity notion has appeared in Quantum Field Theory in seventies of the last century [3]. Few years later, it has been applied to investigation of phase transitions in both classical [4] and quantum [1] lattice spin systems. The Reflection Positivity turned out to be a very useful tool, giving the first rigorous proofs of existence of phase transitions in systems with continuous symmetry group.

The cornerstone of Reflection Positivity for quantum spin systems is the matrix inequality due to Dyson, Lieb and Simon (Lemma 4.1 in [1]). Using this Lemma, authors proved the existence of orderings in the XY as well as Heisenberg models in $d \geq 3$ and for sufficiently small temperature. Later on, this method has been extended to certain class of infinite dimensional operators. This way, the existence of Long-Range Order has been proved for $d \geq 3$ in the system of quantum interacting rotors [5].

Another direction of development of Reflection Positivity techniques was an examination of ground states of quantum spin systems and orderings therein. It turned out that one can take certain zerotemperature limit in the framework of the DLS method. This way, the appearance of Long Range Order has been proved in XY and Heisenberg models in $d=2$ [6], [7, [8]. Later on, it turned out that such a proof can be done directly in the ground state, with the use of another matrix inequality, due to Kennedy, Lieb and Shastry (KLS) [2]. This inequality was further generalized by Schupp [9].

It would be tempting to extend this inequality to infinite-dimensional version, i.e. for certain class of operators. However, to our best knowledge, the operator version of the KLS and Schupp(KLSS) inequalities, suitable for applications to ground states of quantum interacting rotors has not been developed.

This opportunity inspired us to attempts to prove an operator analog of the KLSS inequalities. It turned out to be possible, and this is one of two main results of our paper: extension of the KLSS matrix inequalities to certain class of infinite-dimensional operators. The second group of results which seems to be new are some applications. 
The outline of the paper is as follows. In the Sec. 2 we formulate the operator version of the KLSS inequalities. The application of this inequality is described in the Sec. 3 , it is the proof of the ordering in ground state of $d \geq 2$ rotors (alternative proof to that presented in [10]). The Sec. 4 contains summary, conclusions and description of some open problems.

\section{KLS inequality and its extension for operators}

\subsection{Kennedy, Lieb, Shastry and Schupp matrix inequalities.}

For convenience of the reader, and to show the idea of a proof without operator-theoretic details, we present firstly the matrix version of KLSS inequality.

Theorem 2.1 [2] Let $c, A, B$ be $n \times n$ complex matrices, $|c|:=\sqrt{c^{*} c}$ and $\left|c^{*}\right|:=\sqrt{c c^{*}}$ the moduli of $c$ and $c^{*}$ respectively. Then

$$
\left|\operatorname{Tr} c^{*} B c A^{*}\right| \leq \frac{1}{2}\left[\operatorname{Tr}\left(|c| A|c| A^{*}\right)+\operatorname{Tr}\left(\left|c^{*}\right| B\left|c^{*}\right| B^{*}\right)\right] .
$$

Sketch of the proof: At first let us note that by the polar decomposition theorem $c$ is of the form $c=u|c|$, where $u$ is a partial isometry. Since $u^{*} u|c|=|c|$ and $u|c| u^{*}$ is a positive matrix, the polar decomposition of $c^{*}$ is of the form:

$$
c^{*}=|c| u^{*}=u^{*} u|c| u^{*}=u^{*}\left|c^{*}\right| .
$$

Taking adjoint we get $c=u|c|=\left|c^{*}\right| u$. Therefore

$$
u \sqrt{|c|}=\sqrt{\left|c^{*}\right|} u
$$

according to functional calculus of positive hermitian matrices and

$$
c=\sqrt{\left|c^{*}\right|} u \sqrt{|c|}
$$

Now, let $P$ and $Q$ be matrices introduced by formulae

$$
P=u^{*} \sqrt{\left|c^{*}\right|} B^{*} \sqrt{\left|c^{*}\right|} u, \quad Q=\sqrt{|c|} A^{*} \sqrt{|c|}
$$

Let us remind that the trace functional defines the scalar product on the space of square matrices: $(A \mid B):=\operatorname{Tr} A^{*} B$. Therefore

$$
\left|\operatorname{Tr} P^{*} Q\right| \leq \sqrt{\left(\operatorname{Tr} P^{*} P\right)\left(\operatorname{Tr} Q^{*} Q\right)} \leq \frac{1}{2}\left[\operatorname{Tr} P^{*} P+\operatorname{Tr} Q^{*} Q\right]
$$

due to the Schwarz inequality followed by inequality between geometric mean and arithmetic one. Now using formula (3) and (4) one can easily verify that

$$
P^{*} Q=u^{*} \sqrt{\left|c^{*}\right|} B \sqrt{\left|c^{*}\right|} u \sqrt{|c|} A^{*} \sqrt{|c|}=\sqrt{|c|} u^{*} B c A^{*} \sqrt{|c|}
$$

and due to 2

$$
\operatorname{Tr} P^{*} Q=\operatorname{Tr}\left(\sqrt{|c|} u^{*} B c A^{*} \sqrt{|c|}\right)=\operatorname{Tr}\left(|c| u^{*} B c A^{*}\right)=\operatorname{Tr}\left(c^{*} B c A^{*}\right) .
$$

The module of it coincides with the left hand side of the inequality (1).

In the similar manner we compute the right hand side of (6):

$$
P^{*} P=u^{*} \sqrt{\left|c^{*}\right|} B \sqrt{\left|c^{*}\right|} u u^{*} \sqrt{\left|c^{*}\right|} B^{*} \sqrt{\left|c^{*}\right|} u=u^{*} \sqrt{\left|c^{*}\right|} B\left|c^{*}\right| B^{*} \sqrt{\left|c^{*}\right|} u .
$$

Therefore

$$
\operatorname{Tr} P^{*} P=\operatorname{Tr}\left(u^{*} \sqrt{\left|c^{*}\right|} B\left|c^{*}\right| B^{*} \sqrt{\left|c^{*}\right|} u\right)=\operatorname{Tr}\left(\left|c^{*}\right| B\left|c^{*}\right| B^{*}\right) .
$$

By the similar reasoning we get

$$
\operatorname{Tr} Q^{*} Q=\operatorname{Tr}\left(\sqrt{|c|} A|c| A^{*} \sqrt{|c|}\right)=\operatorname{Tr}\left(|c| A|c| A^{*}\right) .
$$

Combining (7) and (8) we obtain the right hand side of inequality (1). 
Theorem 2.2 9] The KLS inequality (1) holds also for rectangular matrices, i.e. $c$ is $n \times m$ matrix and matricies $A$ and $B$ are $m \times m$ and $n \times n$ respectively.

Proof: It is almost a repetition of the proof for KLS inequality and refers to the modified polar decomposition of the operator $c$ acting between $m$-dimensional and $n$-dimensional spaces. In that situation $|c|,\left|c^{*}\right|$ are positive matrices of dimensions $m \times m$ and $n \times n$, respectively.

\subsection{Operator version of the KLSS inequality}

The main goal of this subsection is to prove a generalized version of the KLSS inequality:

Theorem 2.3 Let $\mathcal{L}$ and $\mathcal{R}$ be separable Hilbert spaces, $A \in \mathcal{B}(\mathcal{L}), \quad B \in \mathcal{B}(\mathcal{R})$ bounded operators acting on $\mathcal{L}$ and $\mathcal{R}$ respectively. Let $c: \mathcal{L} \rightarrow \mathcal{R}$ be a Hilbert-Schmidt operator and $|c|:=\sqrt{c^{*} c}$, and $\left|c^{*}\right|:=\sqrt{c c^{*}}$ be the corresponding moduli. Then

1. $|c|$ and $\left|c^{*}\right|$ are hermitean Hilbert-Schmidt operators acting on $\mathcal{L}$ and $\mathcal{R}$ respectively;

2. $c^{*} B c A^{*}, \quad|c| A|c| A^{*}$ are trace-class operators on $\mathcal{L}$ and $\left|c^{*}\right| B\left|c^{*}\right| B^{*}$ is a trace-class operator on $\mathcal{R}$;

3. the following inequality holds

$$
\left|\operatorname{Tr} c^{*} B c A^{*}\right| \leq \frac{1}{2}\left[\operatorname{Tr}\left(|c| A|c| A^{*}\right)+\operatorname{Tr}\left(\left|c^{*}\right| B\left|c^{*}\right| B^{*}\right)\right]
$$

Remark. In a finite-dimensional case, i.e. $\mathcal{L}=\mathbb{C}^{N}=\mathcal{R}$ the above inequality reduces to the matricial KLS inequality [2] and in more general finite dimensional situation $\operatorname{dim} \mathcal{L} \neq \operatorname{dim} \mathcal{R}$ we obtain the result of Schupp [9].

Proof: To prove our result we shall use some properties of Schatten ideals [17, [18], [19] and now we shall recall necessary results of the theory.

Let $\mathcal{H}$ be a separable Hilbert space, $\mathcal{C B}(\mathcal{H})$ - the set of compact operators on $\mathcal{H}$. For a real number $p \geq 1$ the $p$-Schatten ideal is the set

$$
L^{p}(\mathcal{H}):=\left\{a \in \mathcal{C B}(\mathcal{H}): \operatorname{Tr}\left(|a|^{p}\right)<\infty\right\}
$$

Remark. Sometimes $L^{p}(\mathcal{H})$ is denoted as $\mathcal{J}_{p}$ [14, however, the actual notation corresponding to noncommutative $L^{p}$-spaces seems more natural to us.

For $a \in L^{p}(\mathcal{H})$ let us define:

$$
\|a\|_{p}:=\left(\operatorname{Tr}|a|^{p}\right)^{\frac{1}{p}}
$$

Then it is known that

i) $\left(L^{p}(\mathcal{H}),\|\cdot\|_{p}\right)$ is a Banach space, $L^{p}(\mathcal{H})$ is a two-sided ideal in $\mathcal{B}(\mathcal{H})$, i.e. for any $a \in L^{p}(\mathcal{H})$ and $A, B \in \mathcal{B}(\mathcal{H})$ the operator $A a B \in L^{p}(\mathcal{H})$ and moreover

$$
\|A a B\|_{p} \leq\|A\|\|B\|\|a\|_{p}
$$

ii) if $p, q, r \geq 1$ are such numbers that: $\frac{1}{p}+\frac{1}{q}=\frac{1}{r}$ and $a \in L^{p}(\mathcal{H}), b \in L^{q}(\mathcal{H})$, then $a b \in L^{r}(\mathcal{H})$.

iii) in particular, if $p$ and $q$ satisfy $\frac{1}{p}+\frac{1}{q}=1$ then for $a \in L^{p}(\mathcal{H}), b \in L^{q}(\mathcal{H})$ products $a b, b a \in L^{1}(\mathcal{H})$, $\operatorname{Tr} a b=\operatorname{Tr} b a$ and

$$
|\operatorname{Tr} a b| \leq\|a\|_{p}\|b\|_{q}
$$

Remark. The space $L^{1}(\mathcal{H})$ is the space of trace-class operators on $\mathcal{H}$ and $L^{2}(\mathcal{H})$ is the space of Hilbert-Schmidt class. Clearly $L^{2}(\mathcal{H})$ equipped with the sesquilinear form

$$
L^{2}(\mathcal{H}) \times L^{2}(\mathcal{H}) \ni(a, b) \longmapsto(a \mid b):=\operatorname{Tr} a^{*} b \in \mathbb{C}
$$

is a Hilbert space. 
In what follows we shall also need Hilbert-Schmidt operators in more general settings, namely the operators from one Hilbert space to another.

Definition. An operator $c: \mathcal{L} \rightarrow \mathcal{R}$ is a Hilbert-Schmidt one, if for some orthonormal basis $\{\alpha\}$ in $\mathcal{L}$, the sum

$$
\|c\|_{2}^{2}:=\sum_{\alpha}(c \alpha \mid c \alpha)=\sum_{\alpha}\|c \alpha\|^{2}
$$

is finite.

The set of such operators will be denoted by $L^{2}(\mathcal{L}, \mathcal{R})$. Clearly for $c \in L^{2}(\mathcal{L}, \mathcal{R})$ and any orthonormal basis $\{\beta\}$ in $\mathcal{R}$, we have

$$
\begin{aligned}
& \sum_{\alpha}(c \alpha \mid c \alpha)=\sum_{\alpha}\left(\alpha \mid c^{*} c \alpha\right)=\sum_{\alpha, \beta}|(\beta \mid c \alpha)|^{2} \\
= & \sum_{\alpha, \beta}\left|\left(c^{*} \beta \mid \alpha\right)\right|^{2}=\sum_{\beta}|| c^{*} \beta \|^{2}=\sum_{\beta}\left(\beta \mid c c^{*} \beta\right) .
\end{aligned}
$$

Therefore $c^{*} \in L^{2}(\mathcal{R}, \mathcal{L})$ and the finiteness condition does not depend on the particular choice of an orthonormal basis $\{\alpha\}$. In particular $c^{*} c \in L^{1}(\mathcal{L}), c c^{*} \in L^{1}(\mathcal{R})$ i.e. they are trace-class operators acting on $\mathcal{L}$ and $\mathcal{R}$ respectively. Moreover

$$
\|c\|_{2}^{2}=\left\|c^{*} c\right\|_{1}=\operatorname{Tr}_{\mathcal{L}}\left(c^{*} c\right)=\operatorname{Tr}_{\mathcal{R}}\left(c c^{*}\right)=\left\|c c^{*}\right\|_{1}=\left\|c^{*}\right\|_{2}^{2}
$$

Let us note that for $a, b \in L^{2}(\mathcal{L}, \mathcal{R})$ we have $a^{*} b \in L^{1}(\mathcal{L}), b a^{*} \in L^{1}(\mathcal{R})$. Moreover one can easily check that for any Hilbert spaces $\mathcal{L}^{\prime}, \mathcal{L}, \mathcal{R}, \mathcal{R}^{\prime}$ a modified ideal property holds:

$$
\left(\begin{array}{c}
c \in L^{2}(\mathcal{L}, \mathcal{R}) \\
\text { and } \\
A \in \mathcal{B}\left(\mathcal{L}^{\prime}, \mathcal{L}\right), B \in \mathcal{B}\left(\mathcal{R}, \mathcal{R}^{\prime}\right)
\end{array}\right) \Rightarrow\left(B c A \in L^{2}\left(\mathcal{L}^{\prime}, \mathcal{R}^{\prime}\right)\right) .
$$

As before the space $L^{2}(\mathcal{L}, \mathcal{R})$ forms a Hilbert space equipped with the scalar product

$$
(a \mid b):=\operatorname{Tr}_{\mathcal{L}}\left(a^{*} b\right)=\operatorname{Tr}_{\mathcal{R}}\left(b a^{*}\right)=\left(b^{*} \mid a^{*}\right) .
$$

The last equality can be verified by the similar calculation as above. In what follows to simplify notation the corresponding indices $\mathcal{L}$ or $\mathcal{R}$ will be omitted. As the result, by the Schwarz inequality followed by mean arithmetic-geometric inequality, we obtain

Corollary 2.4 For arbitrary $a, b \in L^{2}(\mathcal{L}, \mathcal{R})$ we have

$$
\left|\operatorname{Tr} a^{*} b\right| \leq \frac{1}{2}\left[\operatorname{Tr} a^{*} a+\operatorname{Tr} b^{*} b\right]
$$

Now we are ready to prove our result. Assume that $c \in L^{2}(\mathcal{L}, \mathcal{R})$. Therefore $c^{*} c$ and $c c^{*}$ are trace-class i.e. $|c| \in L^{2}(\mathcal{L})$ and $\left|c^{*}\right| \in L^{2}(\mathcal{R})$ and this proves the first part of the theorem. The second part easily follows from 12 .

To prove the inequality let us note that by the polar decomposition theorem $c$ is of the form $c=u|c|$ for the unique partial isometry $u \in \mathcal{B}(\mathcal{L}, \mathcal{R}), u:|c|(\mathcal{L}) \rightarrow c(\mathcal{L})$ such that $u^{*} u$ and $u u^{*}$ are projections on the initial and final domain respectively. Now, by uniqueness of the polar decompostion and functional calculus of bounded, self-adjoint operators we obtain (in the same way as for matricies):

$$
u \sqrt{|c|}=\sqrt{\left|c^{*}\right|} u
$$

Therefore

$$
c=\sqrt{\left|c^{*}\right|} u \sqrt{|c|}
$$

Clearly $\sqrt{|c|} \in \mathcal{L}^{4}(\mathcal{L})$ and $\sqrt{\left|c^{*}\right|} \in \mathcal{L}^{4}(\mathcal{R})$ and this observation enables us to follow the proof given for matrices in [2], 9]. As in 5 we define operators $P, Q \in \mathcal{B}(\mathcal{L})$ :

$$
P=u^{*} \sqrt{\left|c^{*}\right|} B^{*} \sqrt{\left|c^{*}\right|} u, \quad Q=\sqrt{|c|} A^{*} \sqrt{|c|}
$$

Remembering that $L^{p}$-spaces are ideals and using property $i i$ ) of Schatten ideals (for $p=q=4$ ) we see that $Q \in L^{2}(\mathcal{L})$ and $\sqrt{\left|c^{*}\right|} B^{*} \sqrt{\left|c^{*}\right|} \in L^{2}(\mathcal{R})$; by 12 we have $P \in L^{2}(\mathcal{L})$. Now 14 reads

$$
\left|\operatorname{Tr} P^{*} Q\right| \leq \frac{1}{2}\left[\operatorname{Tr} P^{*} P+\operatorname{Tr} Q^{*} Q\right]
$$


To compute the left hand side of the above expression let us notice that $u^{*} \sqrt{\left|c^{*}\right|}=\sqrt{|c|} u^{*}$ by formula 15$)$. Using this fact and $(16)$ we get

$$
P^{*} Q=u^{*} \sqrt{\left|c^{*}\right|} B \sqrt{\left|c^{*}\right|} u \sqrt{|c|} A^{*} \sqrt{|c|}=\sqrt{|c|} u^{*} B c A^{*} \sqrt{|c|} .
$$

The operator $u^{*} B c A^{*}$ belongs to $L^{2}(\mathcal{L})$ due to the modified ideal property $\sqrt{12}$. Since $\sqrt{|c|} \in L^{4}(\mathcal{L})$ the operator $\sqrt{|c|} u^{*} B c A^{*} \in L^{\frac{4}{3}}(\mathcal{L})$ by property $\left.i i\right)$ of Schatten ideals. Now using property $\left.i i i\right)$ in the case $p=\frac{4}{3}$ and $q=4$ we have

$$
\operatorname{Tr} P^{*} Q=\operatorname{Tr}\left(\sqrt{|c|} u^{*} B c A^{*} \sqrt{|c|}\right)=\operatorname{Tr}\left(|c| u^{*} B c A^{*}\right)=\operatorname{Tr}\left(c^{*} B c A^{*}\right)
$$

due to (2). The module of it coincides with the left hand side of the inequality (9). In the similar manner we compute the right hand side of (18).

$$
P^{*} P=u^{*} \sqrt{\left|c^{*}\right|} B \sqrt{\left|c^{*}\right|} u u^{*} \sqrt{\left|c^{*}\right|} B^{*} \sqrt{\left|c^{*}\right|} u=u^{*} \sqrt{\left|c^{*}\right|} B\left|c^{*}\right| B^{*} \sqrt{\left|c^{*}\right|} u .
$$

Therefore

$$
\begin{aligned}
\operatorname{Tr} P^{*} P & =\operatorname{Tr}\left(u^{*} \sqrt{\left|c^{*}\right|} B\left|c^{*}\right| B^{*} \sqrt{\left|c^{*}\right|} u\right)=\|\left.\sqrt{\left|c^{*}\right|} B^{*} \sqrt{\left|c^{*}\right|} u\right|_{2} ^{2} \\
& =\left\|u^{*} \sqrt{\left|c^{*}\right|} B \sqrt{\left|c^{*}\right|}\right\|_{2}^{2}=\operatorname{Tr}\left(\sqrt{\left|c^{*}\right|} B^{*} \sqrt{\left|c^{*}\right|} u u^{*} \sqrt{\left|c^{*}\right|} B \sqrt{\left|c^{*}\right|}\right) \\
& =\operatorname{Tr}\left(\sqrt{\left|c^{*}\right|} B^{*}\left|c^{*}\right| B \sqrt{\left|c^{*}\right|}\right)
\end{aligned}
$$

by 11. . Now $\sqrt{\left|c^{*}\right|} B^{*} \in L^{4}(\mathcal{R})$ and $\left|c^{*}\right| B \sqrt{\left|c^{*}\right|} \in L^{\frac{4}{3}}(\mathcal{L})$ and using property $i$ iii) again we obtain

$$
\operatorname{Tr} P^{*} P=\operatorname{Tr}\left(\left|c^{*}\right| B\left|c^{*}\right| B^{*}\right) .
$$

By the similar reasoning we get

$$
\operatorname{Tr} Q^{*} Q=\operatorname{Tr}\left(\sqrt{|c|} A|c| A^{*} \sqrt{|c|}\right)=\operatorname{Tr}\left(|c| A|c| A^{*}\right) .
$$

Combining $(19)$ and 20 we have the right hand side of inequality $(9)$. The proof is done.

\subsection{Main inequality and expectation values}

In this subsection the main inequality $(9)$ will be expressed in terms of expectation values of operators acting on $\mathcal{L} \otimes \mathcal{R}$, where $\mathcal{L}$ and $\mathcal{R}$ are separable, infinite dimensional Hilbert spaces. In that form it will be used in following sections.

Let $\Gamma:=\left\{\psi_{\gamma}\right\}$ denote a fixed orthonormal basis in $\mathcal{L}$. It defines a linear map:

$$
\tilde{\Gamma}: L^{2}(\mathcal{L}, \mathcal{R}) \ni c \mapsto \tilde{\Gamma}(c)=\sum \psi_{\gamma} \otimes c \psi_{\gamma} \in \mathcal{L} \otimes \mathcal{R}
$$

Basic properties of this map are described by

Lemma 2.5 The map $\tilde{\Gamma}$ is unitary, moreover for $B \in \mathcal{B}(\mathcal{R})$ and $a, c \in L^{2}(\mathcal{L}, \mathcal{R})$ we have:

$$
\begin{gathered}
\tilde{\Gamma}(B c)=(I \otimes B) \tilde{\Gamma}(c) \\
(\tilde{\Gamma}(a) \mid(I \otimes B) \tilde{\Gamma}(c))=(\tilde{\Gamma}(a) \mid \tilde{\Gamma}(B c))=\operatorname{Tr}_{\mathcal{L}}\left(a^{*} B c\right)=\operatorname{Tr}_{\mathcal{R}}\left(B c a^{*}\right)=\operatorname{Tr}_{\mathcal{R}}\left(c a^{*} B\right)
\end{gathered}
$$

Proof: It is straightforward to verify that $\tilde{\Gamma}$ is an isometry i.e. for $c_{1}, c_{2} \in L^{2}(\mathcal{L}, \mathcal{R})$ we have $\left(\tilde{\Gamma}\left(c_{1}\right) \mid \tilde{\Gamma}\left(c_{2}\right)\right)=\operatorname{Tr}_{\mathcal{L}}\left(c_{1}^{*} c_{2}\right)$. The formula for $\tilde{\Gamma}(B c)$ is clear. To show unitarity, notice that any $h \in \mathcal{L} \otimes \mathcal{R}$ is of the form $h=\sum \psi_{\gamma} \otimes r_{\gamma}$ for the unique family of vectors $\left(r_{\gamma}\right)$ in $\mathcal{R}$. Let us define a linear map $\tilde{\Gamma}_{1}(h): \mathcal{L} \rightarrow \mathcal{R}$ by $\tilde{\Gamma}_{1}(h) \psi_{\gamma}:=r_{\gamma}$. Then $\tilde{\Gamma}_{1}(h) \in L^{2}(\mathcal{L}, \mathcal{R})$ and simple calculation shows that $\tilde{\Gamma} \tilde{\Gamma}_{1}(h)=h$ and $\tilde{\Gamma}_{1} \tilde{\Gamma}(c)=c$, so $\tilde{\Gamma}$ is unitary and $\tilde{\Gamma}_{1}=\tilde{\Gamma}^{*}$. The formula 21 is also clear; the last two equalities follow from properties of trace: $\operatorname{Tr}_{\mathcal{L}}\left(a^{*} c\right)=\operatorname{Tr}_{\mathcal{R}}\left(c a^{*}\right)$. 
The basis $\Gamma$ defines also an antiunitary involution $J_{\Gamma}: \mathcal{L} \rightarrow \mathcal{L}$ :

$$
J_{\Gamma}\left(\sum k_{\gamma} \psi_{\gamma}\right):=\sum \bar{k}_{\gamma} \psi_{\gamma}, k_{\gamma} \in \mathbb{C} ; \quad J_{\Gamma}^{*}=J_{\Gamma} ; \quad J_{\Gamma}^{2}=I .
$$

Note that for $A \in L^{1}(\mathcal{L})$ we have:

$$
\begin{aligned}
\operatorname{Tr}_{\mathcal{L}}\left(J_{\Gamma} A J_{\Gamma}\right) & =\sum\left(\psi_{\gamma} \mid J_{\Gamma} A J_{\Gamma} \psi_{\gamma}\right)=\sum\left(A J_{\Gamma} \psi_{\gamma} \mid J_{\Gamma} \psi_{\gamma}\right)=\sum\left(A \psi_{\gamma} \mid \psi_{\gamma}\right)= \\
& =\operatorname{Tr}_{\mathcal{L}}\left(A^{*}\right)
\end{aligned}
$$

In the same way for a basis $\Omega=\left\{\phi_{\omega}\right\}$ in $\mathcal{R}$ we have the mapping $\tilde{\Omega}$

$$
\tilde{\Omega}: L^{2}(\mathcal{R}, \mathcal{L}) \ni d \mapsto \tilde{\Omega}(d)=\sum d \phi_{\omega} \otimes \phi_{\omega} \in \mathcal{L} \otimes \mathcal{R}
$$

with the corresponding antiunitary involution $J_{\Omega}: J_{\Omega}\left(\sum l_{\omega} \phi_{\omega}\right):=\sum \overline{l_{\omega}} \phi_{\omega}$, and

Lemma 2.6 The map $\tilde{\Omega}$ is unitary, moreover for $A \in \mathcal{B}(\mathcal{L}), B \in L^{1}(\mathcal{R})$ and $b, d, \in L^{2}(\mathcal{R}, \mathcal{L})$ :

$$
\begin{gathered}
\tilde{\Omega}(A d)=(A \otimes I) \tilde{\Omega}(d) \\
(\tilde{\Omega}(d) \mid(A \otimes I) \tilde{\Omega}(b))=(\tilde{\Omega}(d) \mid \tilde{\Omega}(A b))=\operatorname{Tr}_{\mathcal{R}}\left(d^{*} A b\right)=\operatorname{Tr}_{\mathcal{L}}\left(A b d^{*}\right)=\operatorname{Tr}_{\mathcal{L}}\left(b d^{*} A\right) \\
\operatorname{Tr}_{\mathcal{R}}\left(J_{\Omega} B J_{\Omega}\right)=\operatorname{Tr}_{\mathcal{R}}\left(B^{*}\right)
\end{gathered}
$$

The choice of bases in $\mathcal{L}$ and $\mathcal{R}$ gives us all of these objects and the following straightforward lemma describes relations between both structures:

Lemma 2.7 Let $c \in L^{2}(\mathcal{L}, \mathcal{R})$ and $d \in L^{2}(\mathcal{R}, \mathcal{L})$. Then the following equalities hold:

$$
\begin{aligned}
\tilde{\Gamma}(c) & =\tilde{\Omega}\left(J_{\Gamma} c^{*} J_{\Omega}\right), \quad \tilde{\Gamma}\left(J_{\Omega} d^{*} J_{\Gamma}\right)=\tilde{\Omega}(d) \\
(\tilde{\Omega}(d) \mid \tilde{\Gamma}(c)) & =\left(\tilde{\Omega}(d) \mid \tilde{\Omega}\left(J_{\Gamma} c^{*} J_{\Omega}\right)\right)=\operatorname{Tr}_{\mathcal{R}}\left(d^{*} J_{\Gamma} c^{*} J_{\Omega}\right)= \\
& =\left(\tilde{\Gamma}\left(J_{\Omega} d^{*} J_{\Gamma}\right) \mid \tilde{\Gamma}(c)\right)=\operatorname{Tr}_{\mathcal{L}}\left(J_{\Gamma} d J_{\Omega} c\right)
\end{aligned}
$$

Clearly, the choice of bases $\Gamma, \Omega$ is equivalent to the choice of a basis $\Gamma$ and a unitary operator $U: \mathcal{L} \rightarrow \mathcal{R}$ (the equality $U\left(\psi_{\gamma}\right)=\phi_{\gamma}$ is a definition of operator $U$ or a basis $\Omega=\left\{\phi_{\gamma}\right\}$ ). It is sometimes more convenient to use pair $(\tilde{\Gamma}, U)$ instead of $(\tilde{\Gamma}, \tilde{\Omega})$. In the lemma below we collect formulae we will use:

Lemma 2.8 For $c \in L^{2}(\mathcal{L}, \mathcal{R}), A \in \mathcal{B}(\mathcal{L}), B \in \mathcal{B}(\mathcal{R})$ the following equalities hold:

$$
\begin{aligned}
J_{\Omega} & =U J_{\Gamma} U^{*} \\
(\tilde{\Gamma}(c) \mid(I \otimes B) \tilde{\Gamma}(c)) & =\left(\tilde{\Gamma}\left(\left|c^{*}\right| U\right) \mid(I \otimes B) \tilde{\Gamma}\left(\left|c^{*}\right| U\right)\right) \\
(\tilde{\Gamma}(c) \mid(A \otimes I) \tilde{\Gamma}(c)) & =(\tilde{\Gamma}(U|c|) \mid(A \otimes I) \tilde{\Gamma}(U|c|)) \\
(\tilde{\Gamma}(c) \mid(A \otimes I) \tilde{\Gamma}(c)) & =\left(\tilde{\Gamma}(U|c|) \mid\left(I \otimes U J_{\Gamma} A^{*} J_{\Gamma} U^{*}\right) \tilde{\Gamma}(U|c|)\right) \\
(\tilde{\Gamma}(c) \mid(I \otimes B) \tilde{\Gamma}(c)) & =\left(\tilde{\Gamma}\left(\left|c^{*}\right| U\right) \mid\left(J_{\Gamma} U^{*} B^{*} U J_{\Gamma} \otimes I\right) \tilde{\Gamma}\left(\left|c^{*}\right| U\right)\right) \\
(\tilde{\Gamma}(c) \mid(A \otimes B) \tilde{\Gamma}(c)) & =\operatorname{Tr}_{\mathcal{L}}\left(c^{*} B c J_{\Gamma} A^{*} J_{\Gamma}\right)
\end{aligned}
$$

Proof: For $c \in L^{2}(\mathcal{L}, \mathcal{R})$ recall that $|c|:=\sqrt{c^{*} c} \in L^{2}(\mathcal{L})$ and $\left|c^{*}\right|=\sqrt{c c^{*}} \in L^{2}(\mathcal{R})$; notice also that $U|c| \in L^{2}(\mathcal{L}, \mathcal{R})$ and $\left|c^{*}\right| U \in L^{2}(\mathcal{L}, \mathcal{R})$.

The formula 27 is straightforward, let us prove (28). For $B \in \mathcal{B}(\mathcal{R})$, compute:

$$
\begin{aligned}
(\tilde{\Gamma}(c) \mid(I \otimes B) \tilde{\Gamma}(c)) & =\operatorname{Tr}_{\mathcal{L}}\left(c^{*} B c\right)=\operatorname{Tr}_{\mathcal{R}}\left(c c^{*} B\right)=\operatorname{Tr}_{\mathcal{R}}\left(\left|c^{*}\right|^{2} B\right)=\operatorname{Tr}_{\mathcal{R}}\left(\left|c^{*}\right| B\left|c^{*}\right|\right)= \\
& =\operatorname{Tr}_{\mathcal{L}}\left(U^{*}\left|c^{*}\right| B\left|c^{*}\right| U\right)=\operatorname{Tr}_{\mathcal{L}}\left(\left(\left|c^{*}\right| U\right)^{*} B\left|c^{*}\right| U\right)= \\
& =\left(\tilde{\Gamma}\left(\left|c^{*}\right| U\right) \mid(I \otimes B) \tilde{\Gamma}\left(\left|c^{*}\right| U\right)\right)
\end{aligned}
$$


For the next formula, let $A \in \mathcal{B}(\mathcal{L})$ and using lemma 2.7 we compute:

$$
\begin{aligned}
(\tilde{\Gamma}(c) \mid(A \otimes I) \tilde{\Gamma}(c)) & =\left(\tilde{\Omega}\left(J_{\Gamma} c^{*} J_{\Omega}\right) \mid(A \otimes I) \tilde{\Omega}\left(J_{\Gamma} c^{*} J_{\Omega}\right)\right)=\operatorname{Tr}_{\mathcal{R}}\left(\left(J_{\Gamma} c^{*} J_{\Omega}\right)^{*} A J_{\Gamma} c^{*} J_{\Omega}\right)= \\
& =\operatorname{Tr}_{\mathcal{R}}\left(J_{\Omega} c J_{\Gamma} A\left(J_{\Gamma} c^{*} J_{\Omega}\right)\right)=\operatorname{Tr}_{\mathcal{L}}\left(J_{\Gamma} c^{*} J_{\Omega} J_{\Omega} c J_{\Gamma} A\right)= \\
& =\operatorname{Tr}_{\mathcal{L}}\left(J_{\Gamma}|c|^{2} J_{\Gamma} A\right)
\end{aligned}
$$

Writing the formula above for $U|c|$ instead of $c$ and noting that $|U| c||^{2}=(U|c|)^{*} U|c|=|c|^{2}$ we obtain the equality (29).

We prove the equality $(30)$ :

$$
\begin{aligned}
(\tilde{\Gamma}(c) \mid(A \otimes I) \tilde{\Gamma}(c)) & =\operatorname{Tr}_{\mathcal{L}}\left(J_{\Gamma}|c|^{2} J_{\Gamma} A\right)=\operatorname{Tr}_{\mathcal{L}}\left(\left(J_{\Gamma}|c| J_{\Gamma}\right) J_{\Gamma}|c| J_{\Gamma} A\right)=\operatorname{Tr}_{\mathcal{L}}\left(J_{\Gamma}|c| J_{\Gamma} A J_{\Gamma}|c| J_{\Gamma}\right)= \\
& =\operatorname{Tr}_{\mathcal{L}}\left(|c| J_{\Gamma} A^{*} J_{\Gamma}|c|\right)=\operatorname{Tr}_{\mathcal{L}}\left((U|c|)^{*} U J_{\Gamma} A^{*} J_{\Gamma} U^{*}(U|c|)\right)= \\
& =\left(\tilde{\Gamma}(U|c|) \mid\left(I \otimes U J_{\Gamma} A^{*} J_{\Gamma} U^{*}\right) \tilde{\Gamma}(U|c|)\right)
\end{aligned}
$$

and the formula 31 :

$$
\begin{aligned}
(\tilde{\Gamma}(c) \mid(I \otimes B) \tilde{\Gamma}(c)) & =\operatorname{Tr}_{\mathcal{R}}\left(\left|c^{*}\right| B\left|c^{*}\right|\right)=\operatorname{Tr}_{\mathcal{R}}\left(J_{\Omega}\left|c^{*}\right| B^{*}\left|c^{*}\right| J_{\Omega}\right)= \\
& =\operatorname{Tr}_{\mathcal{R}}\left(J_{\Omega}\left|c^{*}\right| U J_{\Gamma} J_{\Gamma} U^{*} B^{*} U J_{\Gamma} J_{\Gamma} U^{*}\left|c^{*}\right| J_{\Omega}\right)= \\
& =\operatorname{Tr}_{\mathcal{R}}\left(\left(J_{\Gamma} U^{*}\left|c^{*}\right| J_{\Omega}\right)^{*}\left(J_{\Gamma} U^{*} B^{*} U J_{\Gamma}\right)\left(J_{\Gamma} U^{*}\left|c^{*}\right| J_{\Omega}\right)\right)= \\
& =\left(\tilde{\Omega}\left(J_{\Gamma} U^{*}\left|c^{*}\right| J_{\Omega}\right) \mid\left(J_{\Gamma} U^{*} B^{*} U J_{\Gamma} \otimes I\right) \tilde{\Omega}\left(J_{\Gamma} U^{*}\left|c^{*}\right| J_{\Omega}\right)\right)= \\
& =\left(\tilde{\Gamma}\left(\left|c^{*}\right| U\right) \mid\left(J_{\Gamma} U^{*} B^{*} U J_{\Gamma} \otimes I\right) \tilde{\Gamma}\left(\left|c^{*}\right| U\right)\right)
\end{aligned}
$$

Finally, we prove $(32)$ :

$$
\begin{aligned}
(\tilde{\Gamma}(c) \mid(A \otimes B) \tilde{\Gamma}(c)) & =\left(\left(A^{*} \otimes I\right) \tilde{\Gamma}(c) \mid(I \otimes B) \tilde{\Gamma}(c)\right)=\left(\left(A^{*} \otimes I\right) \tilde{\Omega}\left(J_{\Gamma} c^{*} J_{\Omega}\right) \mid \tilde{\Gamma}(B c)\right)= \\
& =\left(\tilde{\Omega}\left(A^{*} J_{\Gamma} c^{*} J_{\Omega}\right) \mid \tilde{\Gamma}(B c)\right)=\operatorname{Tr}_{\mathcal{R}}\left(J_{\Omega} c J_{\Gamma} A J_{\Gamma} c^{*} B^{*} J_{\Omega}\right)= \\
& =\operatorname{Tr}_{\mathcal{R}}\left(B c J_{\Gamma} A^{*} J_{\Gamma} c^{*}\right)=\operatorname{Tr}_{\mathcal{L}}\left(J_{\Gamma} A^{*} J_{\Gamma} c^{*} J_{\Omega} J_{\Omega} B c\right)= \\
& =\operatorname{Tr}_{\mathcal{L}}\left(J_{\Gamma} A^{*} J_{\Gamma} c^{*} B c\right)=\operatorname{Tr}_{\mathcal{L}}\left(c^{*} B c J_{\Gamma} A^{*} J_{\Gamma}\right),
\end{aligned}
$$

where we have used 26 .

Now we want to express the inequality $(9)$ i.e.

$$
2\left|\operatorname{Tr}_{\mathcal{L}}\left(c^{*} B c A^{*}\right)\right| \leq \operatorname{Tr}_{\mathcal{L}}\left(|c| A|c| A^{*}\right)+\operatorname{Tr}_{\mathcal{R}}\left(\left|c^{*}\right| B\left|c^{*}\right| B^{*}\right)
$$

in terms of $\tilde{\Gamma}, J_{\Gamma}, U$ and the scalar product in $\mathcal{L} \otimes \mathcal{R}$.

In the formula $(32)$ we put $B:=U J_{\Gamma} A J_{\Gamma} U^{*}$ and $U|c|$ instead of $c$ and get:

$$
\begin{aligned}
\left(\tilde{\Gamma}(U|c|) \mid\left(A \otimes\left(U J_{\Gamma} A J_{\Gamma} U^{*}\right)\right) \tilde{\Gamma}(U|c|)\right) & =\operatorname{Tr}_{\mathcal{L}}\left(|c| U^{*}\left(U J_{\Gamma} A J_{\Gamma} U^{*}\right)(U|c|) J_{\Gamma} A^{*} J_{\Gamma}\right)= \\
& =\operatorname{Tr}_{\mathcal{L}}\left(|c| J_{\Gamma} A J_{\Gamma}|c| J_{\Gamma} A^{*} J_{\Gamma}\right)
\end{aligned}
$$

Now put into $32 \mid A=U^{*} J_{\Omega} B J_{\Omega} U$ and $\left|c^{*}\right| U$ instead of $c$ and obtain:

$$
\begin{aligned}
\left(\tilde{\Gamma}\left(\left|c^{*}\right| U\right) \mid\left(U^{*} J_{\Omega} B J_{\Omega} U \otimes B\right) \tilde{\Gamma}\left(\left|c^{*}\right| U\right)\right) & =\operatorname{Tr}_{\mathcal{L}}\left(U^{*}\left|c^{*}\right| B\left|c^{*}\right| U J_{\Gamma}\left(U^{*} J_{\Omega} B J_{\Omega} U\right)^{*} J_{\Gamma}\right)= \\
=\operatorname{Tr}_{\mathcal{L}}\left(U^{*}\left|c^{*}\right| B\left|c^{*}\right|\left(U J_{\Gamma} U^{*}\right) J_{\Omega} B^{*} J_{\Omega} U J_{\Gamma}\right) & =\operatorname{Tr}_{\mathcal{L}}\left(U^{*}\left|c^{*}\right| B\left|c^{*}\right|\left(J_{\Omega} J_{\Omega}\right) B^{*} J_{\Omega}\left(J_{\Omega} U\right)\right)= \\
& =\operatorname{Tr}_{\mathcal{L}}\left(U^{*}\left|c^{*}\right| B\left|c^{*}\right| B^{*} U\right)= \\
& =\operatorname{Tr}_{\mathcal{R}}\left(\left|c^{*}\right| B\left|c^{*}\right| B^{*}\right),
\end{aligned}
$$

where (27) i.e. $J_{\Omega}=U J_{\Gamma} U^{*}$ was used.

Finally, writing (33) with $J_{\Gamma} A J_{\Gamma}$ instead of $A$, using (32) and two equalities above we obtain: 
Proposition 2.9 Let $A \in \mathcal{B}(\mathcal{L}), B \in \mathcal{B}(\mathcal{R})$ and $c \in L^{2}(\mathcal{L}, \mathcal{R})$. Then

$$
\begin{aligned}
2|(\tilde{\Gamma}(c) \mid(A \otimes B) \tilde{\Gamma}(c))| \leq( & \left.\tilde{\Gamma}(U|c|) \mid\left(A \otimes U J_{\Gamma} A J_{\Gamma} U^{*}\right) \tilde{\Gamma}(U|c|)\right)+ \\
& +\left(\tilde{\Gamma}\left(\left|c^{*}\right| U\right) \mid\left(U^{*} J_{\Omega} B J_{\Omega} U \otimes B\right) \tilde{\Gamma}\left(\left|c^{*}\right| U\right)\right)
\end{aligned}
$$

We will also need formulae similar to the ones in Lemma 2.8 for some unbounded operators, they are proven in the following proposition.

Proposition 2.10 Let $T$ and $S$ be self-adjoint operators with purely point spectrum acting on $\mathcal{L}$ and $\mathcal{R}$ respectively. Assume that bases $\left\{\psi_{\gamma}\right\}$ and $\left\{\phi_{\gamma}\right\}$ consist of eigenvectors of $T$ and $S: T \psi_{\gamma}=t_{\gamma} \psi_{\gamma}$ and $S \phi_{\gamma}=s_{\gamma} \phi_{\gamma}$; assume moreover that $\tilde{\Gamma}(c) \in D\left((T \otimes I)^{2}\right)$ and $\tilde{\Omega}(d) \in D\left((I \otimes S)^{2}\right)$. Then:

$$
\begin{array}{r}
(\tilde{\Gamma}(c) \mid(T \otimes I) \tilde{\Gamma}(c))=(\tilde{\Gamma}(U|c|) \mid(T \otimes I) \tilde{\Gamma}(U|c|))=\left(\tilde{\Gamma}(U|c|) \mid\left(I \otimes U T U^{*}\right)(\tilde{\Gamma}(U|c|))\right. \\
(\tilde{\Omega}(d) \mid(I \otimes S) \tilde{\Omega}(d))=\left(\tilde{\Omega}\left(U^{*}|d|\right) \mid(I \otimes S) \tilde{\Omega}\left(U^{*}|d|\right)\right)=\left(\tilde{\Omega}\left(U^{*}|d|\right) \mid\left(U^{*} S U \otimes I\right) \tilde{\Omega}\left(U^{*}|d|\right)\right)
\end{array}
$$

Proof: We will prove (35); equalities in (36) can be proven in a similar manner.

Since $\tilde{\Gamma}(c)$ is in the domain of $(T \otimes I)^{2}$ we have:

$$
\begin{aligned}
\left(\tilde{\Gamma}(c) \mid(T \otimes I)^{2} \tilde{\Gamma}(c)\right) & =\sum_{\gamma}\left(\psi_{\gamma} \otimes c \psi_{\gamma} \mid(T \otimes I)^{2} \tilde{\Gamma}(c)\right)=\sum_{\gamma}\left(t_{\gamma}^{2} \psi_{\gamma} \otimes c \psi_{\gamma} \mid \tilde{\Gamma}(c)\right)= \\
& =\sum_{\gamma}\left|t_{\gamma}\right|^{2} \|\left. c \psi_{\gamma}\right|^{2} .
\end{aligned}
$$

This equality means that the series $\sum_{\gamma} t_{\gamma} \psi_{\gamma} \otimes c \psi_{\gamma}$ is convergent. Because $T \otimes I$ is closed and $\psi_{\gamma} \otimes c \psi_{\gamma} \in$ $D(T \otimes I)$ it implies that:

$$
(T \otimes I) \tilde{\Gamma}(c)=\sum_{\gamma} t_{\gamma} \psi_{\gamma} \otimes c \psi_{\gamma}
$$

Since $\left\|U|c| \psi_{\gamma}\right\|^{2}=\left\|c \psi_{\gamma}\right\|^{2}$ the formula $(37)$ implies also convergence of the series $\sum_{\gamma} t_{\gamma} \psi_{\gamma} \otimes U|c| \psi_{\gamma}$ and the equality

$$
(T \otimes I) \tilde{\Gamma}(U|c|)=\sum_{\gamma} t_{\gamma} \psi_{\gamma} \otimes U|c| \psi_{\gamma}
$$

By the lemma 2.7. $\tilde{\Gamma}(U|c|)=\tilde{\Omega}\left(J_{\Gamma}|c| U^{*} J_{\Omega}\right)$. Since $J_{\Gamma}|c| U^{*} \phi_{\gamma} \otimes \phi_{\gamma}=J_{\Gamma}|c| \psi_{\gamma} \otimes \phi_{\gamma} \in D\left(I \otimes U T U^{*}\right)$ the formula (37) means convergence of the series $\sum_{\gamma} t_{\gamma} J_{\Gamma}|c| \psi_{\gamma} \otimes \phi_{\gamma}$ and the equality

$$
\left(I \otimes U T U^{*}\right) \tilde{\Gamma}(U|c|)=\sum_{\gamma} t_{\gamma}\left(J_{\Gamma}|c| \psi_{\gamma}\right) \otimes \phi_{\gamma}
$$

follows. Now combining 38, , 39,, 40$)$ and $\tilde{\Gamma}(U|c|)=\tilde{\Omega}\left(J_{\Gamma}|c| U^{*} J_{\Omega}\right)$ we obtain 35 .

\section{Ground state ordering in the system of 2-dimensional rotors}

\subsection{Description of the system}

Denote by $\Lambda$ the finite subset of the simple cubic lattice in $d$ dimensions: $\Lambda \subset \mathbb{Z}^{d}$. We assume that $\Lambda$ is a (discrete) hypercube and that the number of sites along every edge is even; let us fix $2 N$ to be the length of the hypercube edge:

$$
\Lambda:=\left\{\mathbf{x} \in \mathbb{Z}^{d}:-N+1 \leq x_{i} \leq N, i=1, \ldots, d\right\}
$$

With every site $\mathbf{x} \in \Lambda$ we associate a real variable $\varphi_{\mathbf{x}} \in[0,2 \pi[$. In physical terms, it describes the (angular) position of the rotor at the site $\mathbf{x}$. Equivalently, the position of the rotor at the site $\mathbf{x}$ can be described as a unit vector $\mathbf{s}_{\mathbf{x}} \in \mathbb{S}^{1}$, i.e. one dimensional torus $\mathbb{T}$ :

$$
\mathbf{s}_{\mathbf{x}}=\left[s_{\mathbf{x}}^{x}, s_{\mathbf{x}}^{y}\right]=\left[\cos \varphi_{\mathbf{x}}, \sin \varphi_{\mathbf{x}}\right] .
$$


The total spin is $\mathbf{S}=\sum_{\mathbf{x} \in \Lambda} \mathbf{s}_{\mathbf{x}}$. The Hilbert space $\mathcal{H}_{\mathbf{x}}$ of states on a given site $\mathbf{x}$ is the space of square integrable periodic functions, i.e. $\mathcal{H}_{\mathbf{x}}=L^{2}(\mathbb{T})$. The Hilbert space associated to the whole system is the space of square integrable functions on $|\Lambda|$ - dimensional torus:

$$
\mathcal{H}_{\Lambda}=L^{2}\left(\mathbb{T}^{|\Lambda|}\right)=\underset{\mathbf{x} \in \Lambda}{\otimes} \mathcal{H}_{\mathbf{x}}
$$

The operator $T$ of total kinetic energy of the system of rotors is proportional to the laplacian $\Delta$ :

$$
\mathrm{T}=-\frac{1}{2 I} \sum_{\mathbf{x} \in \Lambda} \frac{\partial^{2}}{\partial \varphi_{\mathbf{x}}^{2}}
$$

where $I>0$ is the moment of inertia of rotor (we assume that all rotors have equal moments of inertia). The system of interacting rotors is defined by the Hamiltonian $H=\mathrm{T}+\hat{V}$, where $\hat{V}$ is an interaction energy between rotors; it is an operator of multiplication by a smooth function $V$. We shall consider the Hamiltonian:

$$
H=\mathrm{T}+\hat{V}_{0}, \quad V_{0}:=-J \sum_{\langle\mathbf{x y}\rangle} \cos \left(\varphi_{\mathbf{x}}-\varphi_{\mathbf{y}}\right)
$$

In this formula $\langle\mathbf{x y}\rangle$ means that $\mathbf{x}$ and $\mathbf{y}$ are the nearest neighbours. By this we mean that all but one coordinates of $\mathbf{x}$ and $\mathbf{y}$ are equal and the ones, say $x_{i}$ and $y_{i}$, that differ satisfy $\left|x_{i}-y_{i}\right|=1 \bmod 2(N-1)$. Thus a site $\mathbf{x}$ laying on the hyperplane defined by $x_{i}=N$ has some of its nearest neighbours on a hyperplanes defined by $x_{i}=-N+1$.

$J$ is the coupling constant: $J>0$ corresponds to ferromagnetic coupling between rotors and $J<0$ to the antiferromagnetic one. In what follows we shall restrict ourselves to the ferromagnetic case, as only in this situation the Reflection Positivity arguments may be applied.

Our Hamiltonian (43) is an elliptic second order differential operator on a $|\Lambda|$-dimensional torus (compact manifold). It is a special case of the more general situation:

Theorem 3.1 [23] Let $(M, g)$ be a compact, oriented, riemannian smooth manifold without boundary; $L: C^{\infty}(M) \rightarrow C^{\infty}(M)$ formally selfadjoint, linear, elliptic, PDO of order $k>0$. Then:

1. L extends uniquely to $\tilde{L}: H^{k}(M) \rightarrow L^{2}(M) ;\left(H^{k}(M)\right.$ is $k$-th Sobolev space)

2. $\tilde{L}$ as an operator on $L^{2}(M)$ (with the domain $H^{k}(M)$ ) is selfadjoint;

3. The spectrum of $\tilde{L}$ consists of isolated eigenvalues of finite multiplicity;

4. Eigenvectors of $\tilde{L}$ are smooth functions.

Let us observe that since $\mathrm{T}$ is positive and $V_{0}$ is a continuous function, the hamiltonian $H$ is bounded from below.

In the following we need in an essential way the uniqueness of the ground state of the Hamiltonian. This is a consequence of positivity improving property of the semigroup $\exp (-t \Delta)$. For convenience of the reader, we recall briefly main definitions and results (we refer to Chapt. XIII of [16] for a detailed presentation).

A non zero function $\Psi \in L^{2}(M)$ is positive iff $\Psi(x) \geq 0$; it is strictly positive if $\Psi(x)>0$ (both inequalities should be understood in almost everywhere sense).

A bounded operator $A$ is:

- positivity preserving if $A \Psi$ is positive for positive $\Psi$;

- positivity improving if $A \Psi$ is strictly positive for positive $\Psi$; equivalent condition is that $(\Psi \mid A \Phi)>0$ for positive $\Psi$ and $\Phi$.

The following is, simplified for our needs, Thm XIII.44 from [16].

Proposition 3.2 Let $H$ be a self adjoint, bounded from below operator on $L^{2}(M)$. Assume the spectrum of $H$ consists of isolated eigenvalues of finite multiplicity. If for every $t>0$ the operator $\exp (-t H)$ is positivity improving then the ground state of $H$ is unique (and strictly positive).

In our situation $M=\mathbb{T}^{|\Lambda|}$, and it is known that for kinetic energy operator $\mathrm{T}$ given by the formula 42 operators $\exp (-t \mathrm{~T}), t>0$ are integral operators; for $\Phi, \Psi \in L^{2}\left(\mathbb{T}^{|\Lambda|}\right)$ :

$$
(\Phi \mid \exp (-t \mathrm{~T}) \Psi)=\int d x d y \overline{\Phi(x)} K(t, x, y) \Psi(y)
$$


The function $K(t, x, y), t>0, x, y \in \mathbb{T}^{|\Lambda|}$ - the heat kernel for $|\Lambda|$ - torus, is explicitely known; it is $a$ strictly positive function, therefore $\exp (-t \mathrm{~T})$ is positivity improving.

The same property for $H=\mathrm{T}+\hat{V}$ holds due to the Trotter product formula (see e.g Chapt. X of [15]):

$$
\exp (-t(\mathrm{~T}+\hat{V})) \Psi=\lim _{n \rightarrow \infty}[\exp (-t \mathrm{~T} / n) \exp (-t \hat{V} / n)]^{n} \Psi
$$

In fact, for any $\Phi, \Psi \in \mathcal{H}_{\Lambda}$ :

$$
(\Phi \mid \exp (-t H) \Psi)=\lim _{n \rightarrow \infty}\left(\Phi \mid[\exp (-t \mathrm{~T} / n) \exp (-t \hat{V} / n)]^{n} \Psi\right)
$$

Let $C:=\sup V$. Since $\exp (-t \mathrm{~T})$ is positivity improving, for strictly positive numbers: $\alpha, \beta, \gamma, \delta>0$ and positive functions $\Phi$ and $\Psi$ we have:

$$
\begin{aligned}
(\Phi \mid \exp (-\alpha \mathrm{T}) \exp (-\beta \hat{V}) \exp (-\gamma \mathrm{T}) \exp (-\delta \hat{V}) \Psi) & \geq e^{-\beta C}(\exp (-\alpha \mathrm{T}) \Phi \mid \exp (-\gamma \mathrm{T}) \exp (-\delta \hat{V}) \Psi) \geq \\
& \geq e^{-(\beta+\delta) C}(\Phi \mid \exp (-(\alpha+\gamma) \mathrm{T}) \Psi)
\end{aligned}
$$

In particular $\left(\Phi \mid[\exp (-t \mathrm{~T} / n) \exp (-t \hat{V} / n)]^{n} \Psi\right) \geq e^{-t C}(\Phi \mid \exp (-t \mathrm{~T}) \Psi)>0$.

Therefore the inequality is preserved in the limit: $(\Phi \mid \exp (-t H) \Psi)>0$ and the uniqueness of the ground state of $H$ follows.

\subsection{Criteria of ordering}

The simplest definition of the order parameter would be an average of the total spin. However, this definition is of little use for the zero field (i.e. as a measure of the spontaneous magnetization) as it is zero due to symmetry. The more physical definition is a zero-field limit of magnetization: $\mathcal{M}=\lim _{h \rightarrow 0} M(h)$ ( $h$ denotes magnetic field) but it is difficult to deal with. More easy to handle is the average of the square of spin. It follows that if the average of the square of spin is different from zero, then the zero-field magnetization is non-zero, too (Griffiths theorem - see [1]). So, we take the average $\left\langle\mathbf{S}^{2}\right\rangle$ as a measure of order parameter. All averages considered in this paper are taken over the ground state. Following this idea, we will prove that if $I$ and $J$ are sufficiently large the ground state of system of interacting rotors, described by the potential (43), exhibits Long-Range Order (LRO):

Theorem 3.3 Assume that $I$ and $J$ satisfy the inequality

$$
\sqrt{I J}>\frac{1}{(2 \pi)^{d}} \int_{[-\pi, \pi]^{d}} \frac{\mathrm{d} \mathbf{k}}{\sqrt{\mathcal{E}(\mathbf{k})}} \equiv \mathcal{I}_{d},
$$

where the function $\mathcal{E}: \mathbb{R}^{d} \rightarrow \mathbb{R}$ is defined by:

$$
\mathcal{E}(\mathbf{k})=d-\sum_{i=1}^{d} \cos k_{i}
$$

Then there exists $C>0$ such that, for sufficiently large $|\Lambda|$ :

$$
\left\langle\left(\frac{\mathbf{S}^{x}}{|\Lambda|}\right)^{2}\right\rangle \equiv\left\langle\left(\frac{1}{|\Lambda|} \sum_{\mathbf{x} \in \Lambda} s_{\mathbf{x}}^{x}\right)^{2}\right\rangle \equiv\left\langle\left(\frac{1}{|\Lambda|} \sum_{\mathbf{x} \in \Lambda} \cos \varphi_{\mathbf{x}}\right)^{2}\right\rangle \geq C
$$

Remarks.

1. The estimation of the form similar to the one in (46) appeared in P-based proofs of LRO in other classes of models including classical and quantum spin systems [1], 4], [5].

2. The integral $\mathcal{I}_{1}$ is divergent, so above theorem does not prove existence of ground-state LRO in $d=1$ but scaling and field-theoretical arguments [12] show (non-rigorously) that there is no ordering in this case.

3. For $d \geq 2$ the integral $\mathcal{I}_{d}$ is finite, so there is LRO in the ground-state. Numerical values of $\mathcal{I}_{d}$ for physical dimensions are: $\mathcal{I}_{2} \approx 0.909173 ; \mathcal{I}_{3} \approx 0.643954$. It indicates the tendency to ordering increases with the growth of a dimension. 
4. For positive temperatures, there is no ordering In dimensions 1 and 2. This statement, analogous to the famous Mermin-Wagner theorem for spin systems [13, has been proven in [5].

5. It is known that in $d \geq 3$, the LRO is present in sufficiently low temperatures, and so in the ground state, too [5. Therefore the Theorem 3.3 is most interesting in $d=2$. Such a result, using another RP arguments, has been proven in [10. In different approach, by scaling and field-theoretical arguments, it was obtained (non-rigorously) in [12].

\subsection{Estimations for basic functions}

To prove the theorem 3.3 it will be convenient to work with Fourier-transformed spins. Let us define:

$$
\hat{s}_{\mathbf{k}}^{\alpha}=\frac{1}{\sqrt{|\Lambda|}} \sum_{\mathbf{x} \in \Lambda} s_{\mathbf{x}}^{\alpha} e^{i \mathbf{k} \cdot \mathbf{x}} \quad(\alpha=x, y)
$$

where $\mathbf{k}$ takes value in the first Brillouin zone, i.e. $k_{j} \in\left\{-\frac{\pi(N-1)}{N}, \ldots, \frac{\pi(N-1)}{N}, \pi\right\}$ for $j=1, \ldots, d$. Let us remark that due to the symmetry of our system we have:

Lemma 3.4 For the system of rotors described by the hamiltonian 43. operators $\hat{s}_{\mathbf{k}}^{\alpha}(\alpha=x, y)$ satisfy identities:

$$
\left\langle\hat{s}_{\mathbf{k}}^{\alpha}\right\rangle=0 \text { and }\left\langle\hat{s}_{\mathbf{k}}^{x}\left(\hat{s}_{\mathbf{k}}^{x}\right)^{*}\right\rangle=\left\langle\hat{s}_{\mathbf{k}}^{y}\left(\hat{s}_{\mathbf{k}}^{y}\right)^{*}\right\rangle .
$$

Proof: Let $R_{\theta}$ be the rotation (in all variables) by $\theta$ :

$$
\left(R_{\theta} f\right)\left(\varphi_{1}, \ldots, \varphi_{|\Lambda|}\right):=f\left(\varphi_{1}-\theta, \ldots, \varphi_{|\Lambda|}-\theta\right)
$$

It is clear that $R_{\theta}$ is unitary. Since the hamiltonian 43 commutes with $R_{\theta}$ and the ground state is unique we have $R_{\theta} \psi_{0}=\lambda \psi_{0}$ for a complex number $\lambda$ with $|\lambda|=1$. Therefore

$$
\langle A\rangle=\left(\psi_{0} \mid A \psi_{0}\right)=\left(R_{\theta} \psi_{0} \mid A R_{\theta} \psi_{0}\right)=\left\langle R_{\theta}^{*} A R_{\theta}\right\rangle
$$

It is easy to check that for any $\mathbf{k}: R_{\pi} \hat{s}_{\mathbf{k}}^{\alpha}=-\hat{s}_{\mathbf{k}}^{\alpha} R_{\pi}$ and $R_{\pi / 2} \hat{s}_{\mathbf{k}}^{x}=\hat{s}_{\mathbf{k}}^{y} R_{\pi / 2}$. Now equalities 48) are clear.

Let us denote by $g_{\mathbf{k}}$ the two-point correlation function in the momentum representation:

$$
g_{\mathbf{k}}=\left\langle\hat{s}_{\mathbf{k}}^{x}\left(\hat{s}_{\mathbf{k}}^{x}\right)^{*}\right\rangle
$$

Clearly $g_{\mathbf{k}} \geq 0$. Since $\hat{s}_{\mathbf{k}}^{x}$ is normal $g_{\mathbf{k}}=\left\langle\left(\hat{s}_{\mathbf{k}}^{x}\right)^{*} \hat{s}_{\mathbf{k}}^{x}\right\rangle$. Remember also that $g_{\mathbf{k}}$ depends on parameters $I$ and $J$. The $g_{\mathbf{k}}$ function and its estimation will play a crucial role in the proof of existence of spontaneous magnetization.

With this notation the inequality (46) can be rewritten as

$$
\frac{1}{|\Lambda|} g_{0} \geq C>0 .
$$

The strategy of the proof of Theorem 3.3 can be described as follows (general ideas are similar to ones in [4], [1], 2]). First (and rather easy) step is the equality

$$
\sum_{\mathbf{k}} g_{\mathbf{k}}=\frac{1}{2}|\Lambda|
$$

Indeed, for the groud state $\psi_{0}$ of $H$ :

$$
\sum_{\mathbf{k}} g_{\mathbf{k}}=\sum_{\mathbf{k}}\left(\hat{s}_{\mathbf{k}}^{x} \psi_{0} \mid \hat{s}_{\mathbf{k}}^{x} \psi_{0}\right)=\sum_{\mathbf{k}, \mathbf{x}, \mathbf{y}} \frac{1}{|\Lambda|}\left(\cos \varphi_{\mathbf{x}} e^{i \mathbf{k x}} \psi_{0} \mid \cos \varphi_{\mathbf{y}} e^{i \mathbf{k y}} \psi_{0}\right)=\sum_{\mathbf{x}}\left(\cos ^{2} \varphi_{\mathbf{x}} \psi_{0} \mid \psi_{0}\right),
$$

where we have used the obvious formula: $\sum_{\mathbf{k}} e^{i \mathbf{k}(\mathbf{x}-\mathbf{y})}=|\Lambda| \delta(\mathbf{x}-\mathbf{y})$. In the same way we obtain: $\sum_{\mathbf{k}}\left(\hat{s}_{\mathbf{k}}^{y} \psi_{0} \mid \hat{s}_{\mathbf{k}}^{y} \psi_{0}\right)=\sum_{\mathbf{x}}\left(\sin ^{2} \varphi_{\mathbf{x}} \psi_{0} \mid \psi_{0}\right)$. Adding these two equalities we get:

$$
\sum_{\mathbf{k}}\left\langle\hat{s}_{\mathbf{k}}^{x}\left(\hat{s}_{\mathbf{k}}^{x}\right)^{*}\right\rangle+\left\langle\hat{s}_{\mathbf{k}}^{y}\left(\hat{s}_{\mathbf{k}}^{y}\right)^{*}\right\rangle=|\Lambda|
$$


and the equality 51 follows due to the second formula of 48

It turns out, that for $\mathbf{k} \neq \mathbf{0}$, and this is the place where the RP arguments and the main inequality (34) is used, the function $g_{\mathbf{k}}$ can be estimated from above by an integrable (for $d>1$ ) function:

$$
g_{\mathbf{k}} \leq \frac{1}{2 \sqrt{I J} \sqrt{\mathcal{E}(\mathbf{k})}}, \quad \mathbf{k} \neq \mathbf{0} .
$$

where the function $\mathcal{E}(\mathbf{k})$ was defined in 45 .

With (51) and (52) in hand we can write

$$
\frac{1}{|\Lambda|} g_{\mathbf{0}}=\frac{1}{2}-\sum_{\mathbf{k} \neq 0} g_{\mathbf{k}} \geq \frac{1}{2 \sqrt{I J}}\left(\sqrt{I J}-\frac{1}{|\Lambda|} \sum_{\mathbf{k} \neq \mathbf{0}} \frac{1}{\sqrt{\mathcal{E}(\mathbf{k})}}\right)
$$

Now the sum $\frac{(2 \pi)^{d}}{|\Lambda|} \sum_{\mathbf{k} \neq \mathbf{0}} \frac{1}{\sqrt{\mathcal{E}(\mathbf{k})}}$ converges as $\Lambda \rightarrow \mathbb{Z}^{d}$ to the integral $\int_{[-\pi, \pi]^{d}} \frac{\mathrm{d} \mathbf{k}}{\sqrt{\mathcal{E}(\mathbf{k})}}$. Therefore the inequality (44) implies that there exists $C>0$ such that for sufficiently large $\Lambda$ we have

$$
\left(\sqrt{I J}-\frac{1}{|\Lambda|} \sum_{\mathbf{k} \neq \mathbf{0}} \frac{1}{\sqrt{\mathcal{E}(\mathbf{k})}}\right) \geq C
$$

i.e the estimate (46). This way to complete the proof of the theorem 3.3 it remains to prove the estimate 52). The existence of such an estimate seems to be a quite general phenomenon, but at present we can prove it only using RP techniques.

The inequality 46 can be viewed as the appearance of the macroscopic occupation of the $\mathbf{k}=\mathbf{0}$ mode. In the other words, it is an indication that in the thermodynamic limit, the $g_{\mathbf{k}}$ function possess non-zero $\delta$ function contribution at $\mathbf{k}=0$.

We will get the inequality (52) by relating $g_{\mathbf{k}}$ to other functions, in particular susceptibility (57), for which we will get an estimate by RP techniques.

Let $\left(\psi_{n}\right)_{n \geq 0}$ be an orthonormal basis consisting of eigenvectors of the Hamiltonian 43 with $H \psi_{n}=$ $E_{n} \psi_{n}$ and $\psi_{0}$ be the ground state. We have:

$$
\begin{aligned}
g_{\mathbf{k}} & =\left\langle\left(\hat{s}_{\mathbf{k}}^{x}\right)^{*} \hat{s}_{\mathbf{k}}^{x}\right\rangle=\left(\hat{s}_{\mathbf{k}}^{x} \psi_{0} \mid \hat{s}_{\mathbf{k}}^{x} \psi_{0}\right)=\sum_{n \geq 0}\left(\hat{s}_{\mathbf{k}}^{x} \psi_{0} \mid \psi_{n}\right)\left(\psi_{n} \mid \hat{s}_{\mathbf{k}}^{x} \psi_{0}\right)= \\
& =\sum_{n>0}\left(\hat{s}_{\mathbf{k}}^{x} \psi_{0} \mid \psi_{n}\right)\left(\psi_{n} \mid \hat{s}_{\mathbf{k}}^{x} \psi_{0}\right)=\sum_{n>0}\left|\left(\hat{s}_{\mathbf{k}}^{x} \psi_{0} \mid \psi_{n}\right)\right|^{2} .
\end{aligned}
$$

There is no term with $n=0$ due to the first formula of 48 . Since $\hat{s}_{\mathbf{k}}^{x}$ is normal $g_{\mathbf{k}}$ can be writtes as:

$$
g_{\mathbf{k}}=\left\langle\hat{s}_{\mathbf{k}}^{x}\left(\hat{s}_{\mathbf{k}}^{x}\right)^{*}\right\rangle=\sum_{n>0}\left|\left(\left(\hat{s}_{\mathbf{k}}^{x}\right)^{*} \psi_{0} \mid \psi_{n}\right)\right|^{2}=\frac{1}{2} \sum_{n>0}\left[\left|\left(\hat{s}_{\mathbf{k}}^{x} \psi_{0} \mid \psi_{n}\right)\right|^{2}+\left|\left(\left(\hat{s}_{\mathbf{k}}^{x}\right)^{*} \psi_{0} \mid \psi_{n}\right)\right|^{2}\right]
$$

Next, we define:

$$
\mathcal{D}_{\mathbf{k}}:=\frac{1}{2}\left\langle\left[\left[\hat{s}_{\mathbf{k}}^{x}, H\right],\left(\hat{s}_{\mathbf{k}}^{x}\right)^{*}\right]\right\rangle
$$

Since $\left(\hat{s}_{\mathbf{k}}^{x}\right) \psi_{0}$ and $\left(\hat{s}_{\mathbf{k}}^{x}\right)^{*} \psi_{0}$ are in the domain of $H$ this definition is correct Short calculation shows that:

$$
\mathcal{D}_{\mathbf{k}}=\frac{1}{2} \sum_{n>0}\left[\left|\left(\hat{s}_{\mathbf{k}}^{x} \psi_{0} \mid \psi_{n}\right)\right|^{2}+\left|\left(\left(\hat{s}_{\mathbf{k}}^{x}\right)^{*} \psi_{0} \mid \psi_{n}\right)\right|^{2}\right]\left(E_{n}-E_{0}\right)
$$

We shall also use the susceptibility which is given by:

$$
\chi_{\mathbf{k}}=\frac{1}{2} \sum_{n>0}\left[\left|\left(\hat{s}_{\mathbf{k}}^{x} \psi_{0} \mid \psi_{n}\right)\right|^{2}+\left|\left(\left(\hat{s}_{\mathbf{k}}^{x}\right)^{*} \psi_{0} \mid \psi_{n}\right)\right|^{2}\right] \frac{1}{E_{n}-E_{0}}
$$

Clearly $\chi_{\mathbf{k}}<\infty$ due to (54) and the fact that $\lim E_{n}=+\infty$.

For a positive integer $n>0$ let

$$
a_{n}:=\sqrt{\left|\left(\hat{s}_{\mathbf{k}}^{x} \psi_{0} \mid \psi_{n}\right)\right|^{2}+\left|\left(\left(\hat{s}_{\mathbf{k}}^{x}\right) * \psi_{0} \mid \psi_{n}\right)\right|^{2}} \frac{1}{\sqrt{E_{n}-E_{0}}},
$$




$$
b_{n}:=\sqrt{\left[\left|\left(\hat{s}_{\mathbf{k}}^{x} \psi_{0} \mid \psi_{n}\right)\right|^{2}+\left|\left(\left(\hat{s}_{\mathbf{k}}^{x}\right)^{*} \psi_{0} \mid \psi_{n}\right)\right|^{2}\right]\left(E_{n}-E_{0}\right)} .
$$

Then $g_{\mathbf{k}}^{2}=\left(\sum a_{n} b_{n}\right)^{2}$ and (by Schwarz inequality):

$$
g_{\mathbf{k}}^{2}=\left(\sum a_{n} b_{n}\right)^{2} \leq\left(\sum a_{n}^{2}\right)\left(\sum b_{n}^{2}\right)=\chi_{\mathbf{k}} \mathcal{D}_{\mathbf{k}}
$$

Notice, that since $\hat{s}_{\mathbf{k}}^{x}$ is a multiplication by a smooth function and $H$ is a second order differential operator, the double commutator appearing in 55 is an operator of multiplication by a smooth function, so it is bounded. In fact by a direct calculation one gets

$$
\mathcal{D}_{\mathbf{k}}=\frac{1}{2}\left\langle\left[\left[\hat{s}_{\mathbf{k}}^{x}, H\right],\left(\hat{s}_{\mathbf{k}}^{x}\right)^{*}\right]\right\rangle \leq \frac{1}{4 I} .
$$

Using this estimate we obtain the inequality:

$$
g_{\mathbf{k}}^{2} \leq \chi_{\mathbf{k}} \cdot \mathcal{D}_{\mathbf{k}} \leq \frac{\chi_{\mathbf{k}}}{4 I}
$$

This shows that an upper bound for $\chi_{\mathbf{k}}$ implies the upper bound for $g_{\mathbf{k}}$.

\subsection{Reflection Positivity arguments}

Let us now proceed along the general line of RP arguments. We perturb the Hamiltonian in the analogous manner as it was done in the case of positive temperatures [5], [10. To do it, let us first modify the original Hamiltonian 43 by a constant (so irrelevant) term introduced by the potential:

$$
V=\frac{J}{2} \sum_{\langle\mathbf{x y}\rangle}\left[\left(\cos \varphi_{\mathbf{x}}-\cos \varphi_{\mathbf{y}}\right)^{2}+\left(\sin \varphi_{\mathbf{x}}-\sin \varphi_{\mathbf{y}}\right)^{2}\right]=V_{0}+J \sum_{\langle\mathbf{x y}\rangle} 1
$$

Now, for a function $b: \Lambda \ni \mathbf{x} \mapsto b_{\mathbf{x}} \in \mathbb{C}$ defined on sites, let us consider the perturbed potential $V(b)$ :

$$
V(b)=\frac{J}{2} \sum_{\langle\mathbf{x y}\rangle}\left[\left|\cos \varphi_{\mathbf{x}}-b_{\mathbf{x}}-\cos \varphi_{\mathbf{y}}+b_{\mathbf{y}}\right|^{2}+\left(\sin \varphi_{\mathbf{x}}-\sin \varphi_{\mathbf{y}}\right)^{2}\right]
$$

and the perturbed hamiltonian

$$
H(b):=\mathrm{T}+\hat{V}(b) .
$$

Let us remark, that if functions $b$ and $b^{\prime}$ differ by a constant function then $H(b)=H\left(b^{\prime}\right)$, in particular for $b$ being a constant $H(b)=H(0)$.

Clearly the perturbed Hamiltonian satisfies all assumptions of Thm 3.1. We will use the Reflection Positivity and the operator inequality in the form given in (34) to prove the following:

Theorem 3.5 Let $E_{0}(b)$ be the ground state energy of the perturbed hamiltonian (62). Then:

$$
E_{0}(b) \geq E_{0}(0)=: E_{0}
$$

The rest of the subsection is devoted to the proof of above theorem. Let us recall the definition of $\Lambda$ (41):

$$
\Lambda:=\left\{\mathbf{x} \in \mathbb{Z}^{d}:-N+1 \leq x_{i} \leq N, i=1, \ldots, d\right\}
$$

We divide the system into two identical subsystems $\Lambda_{L}, \Lambda_{R}$ so that $\Lambda=\Lambda_{L} \cup \Lambda_{R} ; \Lambda_{L}$ is an mirror image of $\Lambda_{R}$ under reflection in the $\Pi$ (hyper) plane given by the equation $x_{1}=\frac{1}{2}$. This way, the subset $\Lambda_{L}$ contains all sites, where the first coordinate is negative or 0 , and $\Lambda_{R}$ - sites, where it is positive.

The Hilbert space $\mathcal{H}_{\Lambda}$ of states of the whole system is a tensor product of two spaces: $\mathcal{H}_{\Lambda}=\mathcal{L} \otimes \mathcal{R}$, where $\mathcal{L}(\mathcal{R})$ is a space of states of subsystem defined on $\Lambda_{L}\left(\Lambda_{R}\right)$.

For a function $b: \Lambda \rightarrow \mathbb{C}$ define:

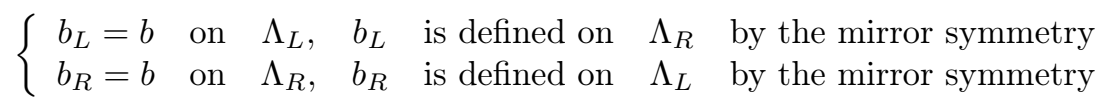

(the standard trick in RP). Our first step to prove inequality 63 is the following : 


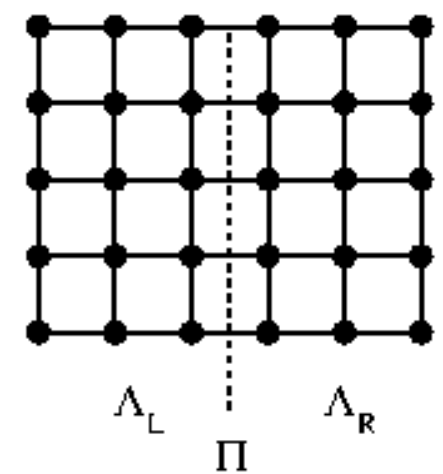

Figure 1: Division of the system into two identical subsystems $\Lambda_{L}, \Lambda_{R}$ by the symmetry plane $\Pi$. The illustration concerns open boundary conditions. Let us stress that in the paper we have to do with periodic boundary conditions, where analogous division can be made.

Lemma 3.6 Let $b: \Lambda \rightarrow \mathbb{C}$ be a function and $b_{L}, b_{R}$ be related to $b$ as in (65). Let hamiltonians $H(b), H\left(b_{R}\right), H\left(b_{L}\right)$ be defined by (62) and $E_{0}(b), E_{0}\left(b_{R}\right), E_{0}\left(b_{L}\right)$ denote their ground state energies respectively. Then:

$$
2 E_{0}(b) \geq E_{0}\left(b_{R}\right)+E_{0}\left(b_{L}\right)
$$

We move the proof of this lemma to the end of this subsection.

Now, we will apply RP arguments to show how the inequality $(66)$ implies $(63)$. For a given function $b: \Lambda \rightarrow \mathbb{C}$ let us call a non-zero bond for $b$ a pair of nearest neighbours $(\mathbf{x}, \mathbf{y})$ with $b(\mathbf{x}) \neq b(\mathbf{y})$. Clearly, if $b \neq$ const then there are $l>0$ non-zero bonds for $b$ and we can choose the symmetry plane, which crosses at least one of them. Notice that if $l_{L}, l_{R}$ are number of non-zero bonds for $b_{L}$ and $b_{R}$ respectively then, we have:

$$
l_{L}+l_{R}<2 l
$$

i.e. at least one of the numbers $\left(l_{L}, l_{R}\right)$ is less than $l$. Therefore for any non-constant $b$ we can pass to a constant one by applying finitely many replacements $b \rightarrow b_{L}$ or $b \rightarrow b_{R}$ (for different symmetry planes). For a given lattice $\Lambda$ the maximal number of steps is bounded independently of $b-$ let $K$ denotes this bound.

Our modified hamiltonian $(62)$ is a positive operator, so the set $\left\{E_{0}(b)\right\}$ is bounded from below and let us define $\mathbf{E}:=\inf \left\{E_{0}(b)\right\}$. From the inequality $(66)$ it follows that for $\epsilon>0$ :

$$
\text { if } \mathbf{E} \leq E_{0}(b) \leq \mathbf{E}+\epsilon \text { then } \mathbf{E} \leq E_{0}\left(b_{L}\right) \leq \mathbf{E}+2 \epsilon \text { and } \mathbf{E} \leq E_{0}\left(b_{R}\right) \leq \mathbf{E}+2 \epsilon
$$

So let $\epsilon>0$ be given. There exists $\tilde{b}$ with $\mathbf{E} \leq E_{0}(\tilde{b}) \leq \mathbf{E}+2^{-K} \epsilon$; replacing succesively $\tilde{b}$ by $\tilde{b}_{L}$ or $\tilde{b}_{R}$ and applying 67 we obtain $\tilde{b}_{0}=$ const with

$$
\mathbf{E} \leq E_{0}\left(\tilde{b}_{0}\right) \leq \mathbf{E}+\epsilon
$$

but since $E_{0}\left(\tilde{b}_{0}\right)=E_{0}(0)=E_{0}$ it follows that $\mathbf{E}=E_{0}-$ so to prove the inequality $(63)$ it remains to prove the lemma 3.6 .

Proof of the lemma 3.6: Consider the perturbed hamiltonian $H(b)=\mathrm{T}+\hat{V}(b)$, where $V(b)$ is given by 62). The proof consists of two steps: the first is rather involved - by using the lemma (2.8) and prop 2.10 we will show that for a smooth function $\Psi \in \mathcal{L} \otimes \mathcal{R}$, there exist $\Psi_{L}, \Psi_{R} \in \mathcal{L} \otimes \mathcal{R}$ such that:

$$
2(\Psi \mid H(b) \Psi) \geq\left(\Psi_{L} \mid H\left(b_{L}\right) \Psi_{L}\right)+\left(\Psi_{R} \mid H\left(b_{R}\right) \Psi_{R}\right)
$$

Since, by the variational principle $\left(\Psi_{L} \mid H\left(b_{L}\right) \Psi_{L}\right) \geq E_{0}\left(b_{L}\right)$ and $\left(\Psi_{R} \mid H\left(b_{R}\right) \Psi_{R}\right) \geq E_{0}\left(b_{R}\right)$, we get:

$$
\left(\Psi_{L} \mid H\left(b_{L}\right) \Psi_{L}\right) \geq E_{0}\left(b_{L}\right)+E_{0}\left(b_{R}\right) .
$$

For $\Psi=\psi_{0}$ - the ground state of $H(b)$, we obtain the inequality 66. 
To simplify notation let us define $a_{\mathbf{x}}(b):=\cos \varphi_{\mathbf{x}}-b_{\mathbf{x}}$; we will write just $a_{\mathbf{x}}$ if it is clear what is $b$. Now, $V(b)$ reads:

$$
V(b)=\frac{J}{2} \sum_{<\mathbf{x y}>}\left|a_{\mathbf{x}}-a_{\mathbf{y}}\right|^{2}+\left(\sin \varphi_{\mathbf{x}}-\sin \varphi_{\mathbf{y}}\right)^{2}
$$

Let us define subsets $B_{L} \subset \Lambda_{L}, B_{R} \subset \Lambda_{R}$ :

$$
\begin{aligned}
& B_{L}:=\left\{\mathbf{x} \in \Lambda_{L}: \text { the first coordinate of } \mathbf{x} \text { is } 0 \text { or }-N+1\right\} \\
& B_{R}:=\left\{\mathbf{x} \in \Lambda_{R}: \text { the first coordinate of } \mathbf{x} \text { is } 1 \text { or } N\right\}
\end{aligned}
$$

Notice that $B_{L}\left(B_{R}\right)$ is the subset of those elements in $\Lambda_{L}\left(\Lambda_{R}\right)$ which have (some of) their nearest neighbours in $\Lambda_{R}\left(\Lambda_{L}\right)$. The potential $V(b)$ can be written as:

$$
\begin{aligned}
V(b) & =\frac{J}{2} \sum_{<\mathbf{x y}>\subset L}\left|a_{\mathbf{x}}-a_{\mathbf{y}}\right|^{2}+\left(\sin \varphi_{\mathbf{x}}-\sin \varphi_{\mathbf{y}}\right)^{2}+\frac{J}{2} \sum_{<\mathbf{x y}>\subset R}\left|a_{\mathbf{x}}-a_{\mathbf{y}}\right|^{2}+\left(\sin \varphi_{\mathbf{x}}-\sin \varphi_{\mathbf{y}}\right)^{2}+ \\
& +\frac{J}{2} \sum_{\mathbf{x} \in B_{L}}\left|a_{\mathbf{x}}-a_{\mathbf{x}^{\prime}}\right|^{2}+\left(\sin \varphi_{\mathbf{x}}-\sin \varphi_{\mathbf{x}^{\prime}}\right)^{2},
\end{aligned}
$$

where $\mathbf{x}^{\prime}$ is the image of $\mathbf{x}$ by the reflection across the hyperplane $\Pi$. The last term of the sum above we write as:

$$
\begin{aligned}
\frac{J}{2} \sum_{\mathbf{x} \in B_{L}}\left|a_{\mathbf{x}}-a_{\mathbf{x}^{\prime}}\right|^{2}+\left(\sin \varphi_{\mathbf{x}}-\sin \varphi_{\mathbf{x}^{\prime}}\right)^{2} & =\frac{J}{2} \sum_{\mathbf{x} \in B_{L}}\left(\left|a_{\mathbf{x}}\right|^{2}+\sin ^{2} \varphi_{\mathbf{x}}\right)+\frac{J}{2} \sum_{\mathbf{x} \in B_{L}}\left(\left|a_{\mathbf{x}^{\prime}}\right|^{2}+\sin ^{2} \varphi_{\mathbf{x}^{\prime}}^{2}\right)+ \\
& -\frac{J}{2} \sum_{\mathbf{x} \in B_{L}}\left(\overline{a_{\mathbf{x}}} a_{\mathbf{x}^{\prime}}+a_{\mathbf{x}} \overline{a_{\mathbf{x}^{\prime}}}+2 \sin \varphi_{\mathbf{x}} \sin \varphi_{\mathbf{x}^{\prime}}\right)
\end{aligned}
$$

Let us define functions:

$$
\begin{gathered}
V_{L}(b):=\frac{J}{2} \sum_{<\mathbf{x y}>\subset L}\left|a_{\mathbf{x}}(b)-a_{\mathbf{y}}(b)\right|^{2}+\left(\sin \varphi_{\mathbf{x}}-\sin \varphi_{\mathbf{y}}\right)^{2}+\frac{J}{2} \sum_{\mathbf{x} \in B_{L}}\left|a_{\mathbf{x}}(b)\right|^{2}+\sin ^{2} \varphi_{\mathbf{x}} \\
V_{R}(b):=\frac{J}{2} \sum_{<\mathbf{x y}>\subset R}\left|a_{\mathbf{x}}(b)-a_{\mathbf{y}}(b)\right|^{2}+\left(\sin \varphi_{\mathbf{x}}-\sin \varphi_{\mathbf{y}}\right)^{2}+\frac{J}{2} \sum_{\mathbf{x} \in B_{L}}\left|a_{\mathbf{x}^{\prime}}(b)\right|^{2}+\sin ^{2} \varphi_{\mathbf{x}^{\prime}} \\
V_{I}(b):=-\frac{J}{2} \sum_{\mathbf{x} \in B_{L}}\left(\overline{a_{\mathbf{x}}} a_{\mathbf{x}^{\prime}}+a_{\mathbf{x}} \overline{a_{\mathbf{x}^{\prime}}}+2 \sin \varphi_{\mathbf{x}} \sin \varphi_{\mathbf{x}^{\prime}}\right)
\end{gathered}
$$

Using above notation we can write the potential $V(b)$ as: $V(b)=V_{L}(b)+V_{R}(b)+V_{I}(b)$ and the corresponding operator $\hat{V}(b)$ as $\hat{V}(b)=\widehat{V_{L}}(b) \otimes I+I \otimes \widehat{V_{R}}(b)+\widehat{V_{I}}(b)$, where

$$
\widehat{V_{I}}(b):=-\frac{J}{2} \sum_{\mathbf{x} \in B_{L}}\left(\overline{a_{\mathbf{x}}} \otimes a_{\mathbf{x}^{\prime}}+a_{\mathbf{x}} \otimes \overline{a_{\mathbf{x}^{\prime}}}+2 \sin \varphi_{\mathbf{x}} \otimes \sin \varphi_{\mathbf{x}^{\prime}}\right) .
$$

For the kinetic term $\mathrm{T}$ we have $\mathrm{T}=\mathrm{T}_{L} \otimes I+I \otimes \mathrm{T}_{R}$.

Now, Let us choose an orthonormal basis $\Gamma:=\left\{\psi_{\gamma}\right\}$ in $\mathcal{L}$ consisting of eigenvectors of $\mathrm{T}_{L}: \mathrm{T}_{L} \psi_{\gamma}=$ $t_{\gamma} \psi_{\gamma}$ and the corresponding involution $J_{\Gamma}$. Note that since $\mathrm{T}_{L}$ is self-adjoint it commutes with $J_{\Gamma}$ :

$$
J_{\Gamma} \mathrm{T}_{L} J_{\Gamma}=\mathrm{T}_{L} .
$$

Let $U: \mathcal{L} \rightarrow \mathcal{R}$ be a unitary operator and $\phi_{\gamma}:=U \psi_{\gamma}$ be the corresponding basis in $\mathcal{R}$.

Let us also assume that

$$
\mathrm{T}_{R}=U \mathrm{~T}_{L} U^{*}
$$

That means, in particular, that $\phi_{\gamma} \in D\left(\mathrm{~T}_{R}\right)$ and $\mathrm{T}_{R} \phi_{\gamma}=t_{\gamma} \phi_{\gamma}$

Let $\Psi=: \tilde{\Gamma}(c) \in \mathcal{L} \otimes \mathcal{R}$ be a smooth function; then it belongs to domains of $\mathrm{T}^{k},\left(\mathrm{~T}_{L} \otimes I\right)^{k},\left(I \otimes \mathrm{T}_{R}\right)^{k}$ for $k=1,2,3, \ldots$ and, using (70), (71) and $(72)$, we can write:

$$
\begin{aligned}
2(\Psi \mid H(b) \Psi) & =2(\Psi \mid \mathrm{T} \Psi)+2(\Psi \mid \widehat{V}(b)) \Psi)= \\
& =\left(\Psi \mid\left(\mathrm{T}_{L} \otimes I\right) \Psi\right)+\left(\Psi \mid\left(\mathrm{T}_{L} \otimes I\right) \Psi\right)+ \\
& +\left(\Psi \mid\left(I \otimes \mathrm{T}_{R}\right) \Psi\right)+\left(\Psi \mid\left(I \otimes \mathrm{T}_{R}\right) \Psi\right)+ \\
& +\left(\Psi \mid\left(\widehat{V_{L}}(b) \otimes I\right) \Psi\right)+\left(\Psi \mid\left(\widehat{V_{L}}(b) \otimes I\right) \Psi\right)+ \\
& +\left(\Psi \mid\left(I \otimes \widehat{V_{R}}(b)\right) \Psi\right)+\left(\Psi \mid\left(I \otimes \widehat{V_{R}}(b)\right) \Psi\right)+ \\
& +2\left(\Psi \mid \widehat{V_{I}}(b) \Psi\right)
\end{aligned}
$$


Now, due to the lemma 2.8 and proposition 2.10 we are going to rewrite various terms appearing in this equality in different form.

By formulae (35) and 75 for terms in $(76)$ we have:

$$
\begin{aligned}
& \left(\Psi \mid\left(\mathrm{T}_{L} \otimes I\right) \Psi\right)=\left(\tilde{\Gamma}(U|c|) \mid\left(\mathrm{T}_{L} \otimes I\right) \tilde{\Gamma}(U|c|)\right) \\
& \left(\Psi \mid\left(\mathrm{T}_{L} \otimes I\right) \Psi\right)=\left(\tilde{\Gamma}(U|c|) \mid\left(I \otimes \mathrm{T}_{R}\right) \tilde{\Gamma}(U|c|)\right)
\end{aligned}
$$

Using (36) with $d=J_{\Gamma} c^{*} J_{\Omega}\left(\right.$ then $|d|=J_{\Omega}\left|c^{*}\right| J_{\Omega}$ and $\left.\tilde{\Omega}\left(U^{*}|d|\right)=\tilde{\Gamma}\left(\left|c^{*}\right| U\right)\right)$ and 75 for terms in 77 :

$$
\begin{aligned}
& \left(\Psi \mid\left(I \otimes \mathrm{T}_{R}\right) \Psi\right)=\left(\tilde{\Gamma}\left(\left|c^{*}\right| U\right) \mid\left(I \otimes \mathrm{T}_{R}\right) \tilde{\Gamma}\left(\left|c^{*}\right| U\right)\right) \\
& \left(\Psi \mid\left(I \otimes \mathrm{T}_{R}\right) \Psi\right)=\left(\tilde{\Gamma}\left(\left|c^{*}\right| U\right) \mid\left(\mathrm{T}_{L} \otimes I\right) \tilde{\Gamma}\left(\left|c^{*}\right| U\right)\right)
\end{aligned}
$$

For terms appearing in $(78)$ and $(79)$ by the use of $(29),(30), 28)$ and $(31)$ we get:

$$
\begin{aligned}
\left(\Psi \mid\left(\widehat{V_{L}}(b) \otimes I\right) \Psi\right) & =\left(\tilde{\Gamma}(U|c|) \mid\left(\widehat{V_{L}}(b) \otimes I\right) \tilde{\Gamma}(U|c|)\right) \\
\left(\Psi \mid\left(\widehat{V_{L}}(b) \otimes I\right) \Psi\right) & =\left(\tilde{\Gamma}(U|c|) \mid\left(I \otimes U J_{\Gamma}\left(\widehat{V_{L}}(b)\right)^{*} J_{\Gamma} U^{*}\right) \tilde{\Gamma}(U|c|)\right) \\
\left(\Psi \mid\left(I \otimes \widehat{V_{R}}(b)\right) \Psi\right) & =\left(\tilde{\Gamma}\left(\left|c^{*}\right| U\right) \mid\left(I \otimes \widehat{V_{R}}(b)\right) \tilde{\Gamma}\left(\left|c^{*}\right| U\right)\right) \\
\left(\Psi \mid\left(I \otimes \widehat{V_{R}}(b)\right) \Psi\right) & =\left(\tilde{\Gamma}\left(\left|c^{*}\right| U\right) \mid\left(U^{*} J_{\Omega}\left(\widehat{V_{R}}(b)\right)^{*} J_{\Omega} U \otimes I\right) \tilde{\Gamma}\left(\left|c^{*}\right| U\right)\right)
\end{aligned}
$$

Now we are going to use the inequality (34) to estimate the term in 80 .

Since $\left(\Psi \mid\left(\overline{a_{\mathbf{x}}} \otimes a_{\mathbf{x}^{\prime}}+a_{\mathbf{x}} \otimes \overline{a_{\mathbf{x}^{\prime}}}\right) \Psi\right)$ is real we have:

$$
\begin{aligned}
\left(\Psi \mid\left(\overline{a_{\mathbf{x}}} \otimes a_{\mathbf{x}^{\prime}}+a_{\mathbf{x}} \otimes \overline{a_{\mathbf{x}^{\prime}}}\right) \Psi\right) & \leq\left|\left(\Psi \mid\left(\overline{a_{\mathbf{x}}} \otimes a_{\mathbf{x}^{\prime}}+a_{\mathbf{x}} \otimes \overline{a_{\mathbf{x}^{\prime}}}\right) \Psi\right)\right| \leq \\
& \leq\left|\left(\Psi \mid\left(\overline{a_{\mathbf{x}}} \otimes a_{\mathbf{x}^{\prime}}\right) \Psi\right)\right|+\left|\left(\Psi \mid\left(a_{\mathbf{x}} \otimes \overline{a_{\mathbf{x}^{\prime}}}\right) \Psi\right)\right|
\end{aligned}
$$

Applying the inequality 34 to each of two terms we get:

$$
\begin{aligned}
2\left|\left(\Psi \mid\left(\overline{a_{\mathbf{x}}} \otimes a_{\mathbf{x}^{\prime}}\right) \Psi\right)\right| & \leq\left(\tilde{\Gamma}(U|c|) \mid\left(\overline{a_{\mathbf{x}}} \otimes U J_{\Gamma} \overline{a_{\mathbf{x}}} J_{\Gamma} U^{*}\right) \tilde{\Gamma}(U|c|)\right)+ \\
& +\left(\tilde{\Gamma}\left(\left|c^{*}\right| U\right) \mid\left(U^{*} J_{\Omega} a_{\mathbf{x}^{\prime}} J_{\Omega} U \otimes a_{\mathbf{x}^{\prime}}\right) \tilde{\Gamma}\left(\left|c^{*}\right| U\right)\right)
\end{aligned}
$$

and

$$
\begin{aligned}
2\left|\left(\Psi \mid\left(a_{\mathbf{x}} \otimes \overline{a_{\mathbf{x}^{\prime}}}\right) \Psi\right)\right| & \leq\left(\tilde{\Gamma}(U|c|) \mid\left(a_{\mathbf{x}} \otimes U J_{\Gamma} a_{\mathbf{x}} J_{\Gamma} U^{*}\right) \tilde{\Gamma}(U|c|)\right)+ \\
& +\left(\tilde{\Gamma}\left(\left|c^{*}\right| U\right) \mid\left(U^{*} J_{\Omega} \overline{a_{\mathbf{x}^{\prime}}} J_{\Omega} U \otimes \overline{a_{\mathbf{x}^{\prime}}}\right) \tilde{\Gamma}\left(\left|c^{*}\right| U\right)\right)
\end{aligned}
$$

Similarly, since $2\left(\Psi \mid\left(\sin \varphi_{\mathbf{x}} \otimes \sin \varphi_{\mathbf{x}^{\prime}}\right) \Psi\right)$ is real:

$$
\begin{aligned}
2\left(\Psi \mid\left(\sin \varphi_{\mathbf{x}} \otimes \sin \varphi_{\mathbf{x}^{\prime}}\right) \Psi\right) & \leq 2\left|\left(\Psi \mid\left(\sin \varphi_{\mathbf{x}} \otimes \sin \varphi_{\mathbf{x}^{\prime}}\right) \Psi\right)\right| \leq \\
& \leq\left(\tilde{\Gamma}(U|c|) \mid\left(\sin \varphi_{\mathbf{x}} \otimes U J_{\Gamma} \sin \varphi_{\mathbf{x}} J_{\Gamma} U^{*}\right) \tilde{\Gamma}(U|c|)\right)+ \\
& +\left(\tilde{\Gamma}\left(\left|c^{*}\right| U\right) \mid\left(U^{*} J_{\Omega} \sin \varphi_{\mathbf{x}^{\prime}} J_{\Omega} U \otimes \sin \varphi_{\mathbf{x}^{\prime}}\right) \tilde{\Gamma}\left(\left|c^{*}\right| U\right)\right)
\end{aligned}
$$

Adding these three inequalities and multiplting by a negative number $(-J / 2)$ we obtain the estimate for 80 :

$$
2\left(\Psi \mid \widehat{V_{I}}(b) \Psi\right) \geq\left(\tilde{\Gamma}(U|c|) \mid K_{L} \tilde{\Gamma}(U|c|)\right)+\left(\tilde{\Gamma}\left(\left|c^{*}\right| U\right) \mid K_{R} \tilde{\Gamma}\left(\left|c^{*}\right| U\right)\right),
$$

where operators $K_{L}$ and $K_{R}$ are defined by:

$$
\begin{gathered}
K_{L}:=-\frac{J}{2} \sum_{\mathbf{x} \in B_{L}}\left[\overline{a_{\mathbf{x}}} \otimes U J_{\Gamma} \overline{a_{\mathbf{x}}} J_{\Gamma} U^{*}+a_{\mathbf{x}} \otimes U J_{\Gamma} a_{\mathbf{x}} J_{\Gamma} U^{*}+2 \sin \varphi_{\mathbf{x}} \otimes U J_{\Gamma} \sin \varphi_{\mathbf{x}} J_{\Gamma} U^{*}\right] \\
K_{R}:=-\frac{J}{2} \sum_{\mathbf{x} \in B_{L}}\left[U^{*} J_{\Omega} a_{\mathbf{x}^{\prime}} J_{\Omega} U \otimes a_{\mathbf{x}^{\prime}}+U^{*} J_{\Omega} \overline{a_{\mathbf{x}^{\prime}}} J_{\Omega} U \otimes \overline{a_{\mathbf{x}^{\prime}}}+2 U^{*} J_{\Omega} \sin \varphi_{\mathbf{x}^{\prime}} J_{\Omega} U \otimes \sin \varphi_{\mathbf{x}^{\prime}}\right]
\end{gathered}
$$


Putting all together we obtain the following inequality :

$$
\begin{array}{r}
2(\Psi \mid H(b) \Psi) \geq\left(\Psi_{L} \mid H_{1} \Psi_{L}\right)+\left(\Psi_{R} \mid H_{2} \Psi_{R}\right), \text { where } \\
\Psi_{L} ;=\tilde{\Gamma}(U|c|), \Psi_{R}:=\tilde{\Gamma}\left(\left|c^{*}\right| U\right) \\
H_{1}:=\mathrm{T}_{L} \otimes I+I \otimes \mathrm{T}_{R}+\widehat{V_{L}}(b) \otimes I+I \otimes U J_{\Gamma}\left(\widehat{V_{L}}(b)\right)^{*} J_{\Gamma} U^{*}+K_{L} \\
H_{2}:=\mathrm{T}_{L} \otimes I+I \otimes \mathrm{T}_{R}+U^{*} J_{\Omega}\left(\widehat{V_{R}}(b)\right)^{*} J_{\Omega} U \otimes I+I \otimes \widehat{V_{R}}(b)+K_{R}
\end{array}
$$

We would like to have $H_{1}=H\left(b_{L}\right), H_{2}=H\left(b_{R}\right)$. Notice that the $H_{1}$ and $H_{2}$ depend only on composition $U J_{\Gamma}$ (by (27) $J_{\Omega} U=U J_{\Gamma}$ ); remember also that we have assumed in 75 that $\mathrm{T}_{R}=$ $U \mathrm{~T}_{L} U^{*}$

Let $M: \Lambda \rightarrow \Lambda$ be the reflection across our (hyper)plane $\Pi$ and let $U_{M}: \mathcal{L} \rightarrow \mathcal{R}$ be the unitary defined by

$$
\begin{gathered}
\left(U_{M} \psi\right)\left(\varphi_{\mathbf{y}_{1}}, \varphi_{\mathbf{y}_{2}}, \ldots\right):=\psi\left(\varphi_{\mathbf{x}_{1}}, \varphi_{\mathbf{x}_{2}}, \ldots\right) \\
\mathbf{y}_{i}:=M\left(\mathbf{x}_{i}\right), \mathbf{x}_{i} \in \Lambda_{L}, i=1, \ldots,\left|\Lambda_{L}\right|
\end{gathered}
$$

and $J_{0}$ be the complex conjugation (we will use $J_{0}$ for conjugations on $\mathcal{L}$ and $\mathcal{R}$ ). It is clear that

$$
J_{0} U_{M}=U_{M} J_{0}, \mathrm{~T}_{L} J_{0}=J_{0} \mathrm{~T}_{L}, \mathrm{~T}_{R} J_{0}=J_{0} \mathrm{~T}_{R},
$$

Define the unitary $U:=J_{0} U_{M} J_{\Gamma}=U_{M} J_{0} J_{\Gamma}$; then

$$
U \mathrm{~T}_{L} U^{*}=U_{M} J_{0} J_{\Gamma} \mathrm{T}_{L} J_{\Gamma} J_{0} U_{M}^{*}=U_{M} \mathrm{~T}_{L} U_{M}^{*}=\mathrm{T}_{R} \text { and } U J_{\Gamma}=J_{0} U_{M}=U_{M} J_{0} .
$$

We will analyze $H_{1}$. Clearly we have $V_{L}(b)=V_{L}\left(b_{L}\right)$; since the function $V_{L}(b)$ is real $\left(\widehat{V_{L}}(b)\right)^{*}=\widehat{V_{L}}(b)$ and $J_{0} \widehat{V_{L}}(b) J_{0}=\widehat{V_{L}}(b)$. Therefore

$$
U J_{\Gamma}\left(\widehat{V_{L}}(b)\right)^{*} J_{\Gamma} U^{*}=U_{M} \widehat{V_{L}}(b) U_{M}^{*}=\widehat{V_{R}}\left(b_{L}\right)
$$

This way we obtain:

$$
H_{1}=\mathrm{T}+\widehat{V_{L}}\left(b_{L}\right) \otimes I+I \otimes \widehat{V_{R}}\left(b_{L}\right)+K_{L}
$$

Let us show that $K_{L}$ given by 81 is equal $\widehat{V}_{I}\left(b_{L}\right)$ defined in 73 :

$$
\begin{aligned}
U J_{\Gamma} \overline{a_{\mathbf{x}}(b)} J_{\Gamma} U^{*} & =U_{M} J_{0} \overline{\left(\cos \varphi_{\mathbf{x}}-b_{\mathbf{x}}\right)} J_{0} U_{M}^{*}=U_{M}\left(\cos \varphi_{\mathbf{x}}-b_{\mathbf{x}}\right) U_{M}=\cos \varphi_{\mathbf{x}^{\prime}}-b_{L}\left(\mathbf{x}^{\prime}\right)= \\
& =a_{\mathbf{x}^{\prime}}\left(b_{L}\right),
\end{aligned}
$$

where $\mathbf{x}^{\prime}:=M(\mathbf{x})$ (because for $\mathbf{x} \in \Lambda_{L} b(\mathbf{x})=b_{L}(\mathbf{x})=b_{L}\left(\mathbf{x}^{\prime}\right)$ ); the next term:

$$
U J_{\Gamma} a_{\mathbf{x}}(b) J_{\Gamma} U^{*}=U_{M} \overline{a_{\mathbf{x}}(b)} U_{M}^{*}=\overline{a_{\mathbf{x}^{\prime}}\left(b_{L}\right)}
$$

and finally

$$
U J_{\Gamma} \sin \varphi_{\mathbf{x}} J_{\Gamma} U^{*}=U_{M} \sin \varphi_{\mathbf{x}} U_{M}^{*}=\sin \varphi_{\mathbf{x}^{\prime}}
$$

So we get:

$$
K_{L}=-\frac{J}{2} \sum_{\mathbf{x} \in B_{L}}\left[\overline{a_{\mathbf{x}}\left(b_{L}\right)} \otimes a_{\mathbf{x}^{\prime}}\left(b_{L}\right)+a_{\mathbf{x}}\left(b_{L}\right) \otimes \overline{a_{\mathbf{x}^{\prime}}\left(b_{L}\right)}+2 \sin \varphi_{\mathbf{x}} \otimes \sin \varphi_{\mathbf{x}^{\prime}}\right]
$$

and this is $\widehat{V_{I}}\left(b_{L}\right)$, so really $H_{1}=H\left(b_{L}\right)$; in the same way one gets $H_{2}=H\left(b_{R}\right)$.

This way the proof of the inequality (68) and the lemma 3.6 is completed as well as the proof of the inequality 63 .

\subsection{Estimations giving LRO}

In this subsection we finally complete the proof of LRO, i.e. we show the inequality (52). The inequality 63 implies that

$$
\left.\frac{\mathrm{d}^{2} E(\lambda b)}{\mathrm{d} \lambda^{2}}\right|_{\lambda=0} \geq 0
$$


for an arbitrary $b$. It turns out that if we take $b$ being plane wave with the wave vector $\mathbf{k}$ then we get (52). Let us present calculations in more details.

For the moment, let us keep the $b$ function being arbitrary. Write the perturbed Hamiltonian 62 with the $b$ function rescaled by a factor $\lambda \in \mathbb{R}$ :

$$
H(\lambda b)=H(0)+\lambda H^{\prime}(b)+\lambda^{2} C(b)
$$

where

$$
H^{\prime}(b):=-J R e\left(\sum_{<\mathbf{x y}>}\left(\cos \varphi_{\mathbf{x}}-\cos \varphi_{\mathbf{y}}\right)\left(b_{\mathbf{x}}-b_{\mathbf{y}}\right)\right), C(b):=\frac{J}{2} \sum_{<\mathbf{x y}>}\left|b_{\mathbf{x}}-b_{\mathbf{y}}\right|^{2}
$$

Let $E_{0}(\lambda b)$ be the ground state energy of the operator $H(\lambda b)$, and $\stackrel{(2)}{\Delta} E_{0}(b)$ - the correction to ground state energy in the second order perturbation theory for the Hamiltonian $H(0)+\lambda H^{\prime}(b)$, i.e.

$$
\stackrel{(2)}{\Delta} E_{0}(b)=\sum_{n>0} \frac{\left|\left(\psi_{n} \mid H^{\prime}(b) \psi_{0}\right)\right|^{2}}{E_{0}-E_{n}}
$$

Therefore:

$$
\left.\frac{\mathrm{d}^{2} E(\lambda b)}{\mathrm{d} \lambda^{2}}\right|_{\lambda=0}=\stackrel{(2)}{\Delta} E_{0}(b)+2 C(b) \geq 0
$$

Choose now the $b$ function as

$$
\mathrm{b}_{\mathbf{x}}=\frac{1}{\sqrt{|\Lambda|}} e^{i \mathbf{k} \cdot \mathbf{x}}
$$

With such a choice $(91)$ reads:

$$
C(b):=\frac{J}{2|\Lambda|} \sum_{<\mathbf{x y}>}\left|e^{i \mathbf{k} \cdot \mathbf{x}}-e^{i \mathbf{k} \cdot \mathbf{y}}\right|^{2}=J \mathcal{E}(\mathbf{k})
$$

and, since

$$
\begin{gathered}
\frac{1}{\sqrt{|\Lambda|}} \sum_{\langle\mathbf{x y}\rangle}\left(\cos \varphi_{\mathbf{x}}-\cos \varphi_{\mathbf{y}}\right)\left(e^{i \mathbf{k} \cdot \mathbf{x}}-e^{i \mathbf{k} \cdot \mathbf{y}}\right)=s_{\mathbf{k}}^{x} \sum_{j=1}^{d}\left(2-2 \cos \mathbf{k}_{j}\right) \equiv 2 s_{\mathbf{k}}^{x} \mathcal{E}(\mathbf{k}) \\
H^{\prime}(b)=-2 \operatorname{JE}(\mathbf{k}) \operatorname{Re}\left(s_{\mathbf{k}}^{x}\right)
\end{gathered}
$$

Therefore by $(92)$ :

$$
-4 J^{2} \mathcal{E}^{2}(\mathbf{k}) \sum_{n>0} \frac{\left|\left(\psi_{n} \mid \operatorname{Re}\left(s_{\mathbf{k}}^{x}\right) \psi_{0}\right)\right|^{2}}{E_{n}-E_{0}}+2 J \mathcal{E}(\mathbf{k}) \geq 0
$$

By the similar computations, replacing $b$ by $i b$ we obtain: $H^{\prime}(i b)=2 J \mathcal{E}(\mathbf{k}) \operatorname{Im}\left(s_{\mathbf{k}}^{x}\right)$ and

$$
-4 J^{2} \mathcal{E}^{2}(\mathbf{k}) \sum_{n>0} \frac{\left|\left(\psi_{n} \mid \operatorname{Im}\left(s_{\mathbf{k}}^{x}\right) \psi_{0}\right)\right|^{2}}{E_{n}-E_{0}}+2 J \mathcal{E}(\mathbf{k}) \geq 0
$$

Adding these inequalities we get (remember $J \mathcal{E}(\mathbf{k}) \geq 0$ ):

$$
\sum_{n>0} \frac{\left|\left(\psi_{n} \mid \operatorname{Re}\left(s_{\mathbf{k}}^{x}\right) \psi_{0}\right)\right|^{2}}{E_{n}-E_{0}}+\frac{\left|\left(\psi_{n} \mid \operatorname{Im}\left(s_{\mathbf{k}}^{x}\right) \psi_{0}\right)\right|^{2}}{E_{n}-E_{0}} \leq \frac{1}{J \mathcal{E}(\mathbf{k})}
$$

For complex numbers $\alpha:=\left(\psi_{n} \mid s_{\mathbf{k}}^{x} \psi_{0}\right)$ and $\beta:=\left(\psi_{n} \mid\left(s_{\mathbf{k}}^{x}\right)^{*} \psi_{0}\right)$, by the paralleogram law : $|\alpha+\beta|^{2}+$ $|\alpha-\beta|^{2}=2\left(|\alpha|^{2}+|\beta|^{2}\right.$, we get

$$
\left|\left(\psi_{n} \mid \operatorname{Re}\left(s_{\mathbf{k}}^{x}\right) \psi_{0}\right)\right|^{2}+\left|\left(\psi_{n} \mid \operatorname{Im}\left(s_{\mathbf{k}}^{x}\right) \psi_{0}\right)\right|^{2}=\frac{1}{2}\left(\left.\left(\psi_{n} \mid s_{\mathbf{k}}^{x} \psi_{0}\right)\right|^{2}+\left|\left(\psi_{n} \mid\left(s_{\mathbf{k}}^{x}\right)^{*} \psi_{0}\right)\right|^{2}\right),
$$

and finally:

$$
\frac{1}{2} \sum_{n>0} \frac{\left|\left(\psi_{n} \mid s_{\mathbf{k}}^{x} \psi_{0}\right)\right|^{2}}{E_{n}-E_{0}}+\frac{\left|\left(\psi_{n} \mid\left(s_{\mathbf{k}}^{x}\right)^{*} \psi_{0}\right)\right|^{2}}{E_{n}-E_{0}} \leq \frac{1}{J \mathcal{E}(\mathbf{k})}
$$


The LHS of this inequality is just succeptibility $\chi_{\mathbf{k}}$, compare $\sqrt[57]{57}$, so we have $\chi_{\mathbf{k}} \leq \frac{1}{J \mathcal{E}(\mathbf{k})}$. Using 58 and (59), we obtain the estimate (52):

$$
g_{\mathbf{k}}^{2} \leq \chi_{\mathbf{k}} \cdot \mathcal{D}_{\mathbf{k}} \leq \chi_{\mathbf{k}} \frac{1}{4 I} \leq \frac{1}{4 I J \mathcal{E}(\mathbf{k})}
$$

The proof of theorem 3.3 is complete.

\section{Summary}

We have extended the Kennedy-Lieb-Shastry-Schupp matrix inequality to the case where matrices are replaced by certain infinite dimensional operators. Similar result has been proven in [5] for another matrix inequality - the DLS lemma, which is crucial for the proof of occurrence of LRO in the system of interacting rotors in low temperatures in $d \geq 3$.

With the use of this inequality and Reflection Positivity technology, we have formulated sufficient condition (44) for ordering in the ground state of the system of interacting rotors. In particular, the LRO is present in $d \geq 2$ for sufficiently large value of $I J$. This way, we have shown the occurrence of the LRO in the ground state of interacting rotor systems in a direct manner. In the paper [10, analogous result has been proven by Reflection Positivity technique, but without checking some assumptions (validity of certain limiting procedure). Our present approach does not suffer from this drawback. This result has also been obtained in non-rigorous way by scaling and field-theoretic arguments [12]

We are convinced that our result can be extended to other rotor systems: other (bipartite) lattices and larger space of internal degrees of freedom, for instance, for $O(n)$ systems.

One can pose the problem concerning the occurrence the ordering in opposite situation, i.e. for quantity $I J$ being small. To our best knowledge, this is an open question. One can suspect that the LRO should be absent. Such expectation is motivated by the paper [21], where somewhat similar result has been proved: There is no ordering in the anharmonic crystal model provided mass of the oscilator is sufficiently small.

There are numerous interesting rotor-like systems, which do not fulfill conditions allowing an application of Reflection Positivity techniques. One of most important of them, is the lattice system of interacting bosons (for instance, the Bose Hubbard model). The Hamiltonian of this system, written in the language of coherent states, becomes the Hamiltonian of interacting rotors of the form (43), plus one term more (see, for instance [22]). This last term spoils the Reflection Positivity, an it seems to be not possible to apply these techniques to the analysis of interacting boson systems. (Only exception is the paper [20], where the Bose-Einstein condensation has been proved for hard-core bosons on optical lattice. Here, the term spoiling RP is absent due to the hard-core condition). Here we tackle with the long-standing and important problem: How to extend the range of applicability of Reflection Positivity technique, which works for certain problems, and does not work for apparently very similar ones.

Another interesting problem is the occurrence of the Kosterlitz-Thouless transition [24] in the twodimensional rotor system. On physical grounds, one can expect occurrence of this transition, at least for large momentum of inertia (the quantum-mechanical rotors should not differ too much from the 2d XY model, for which such a transition has been rigorously proven [25]) However, we are not aware on rigorous results for interacting rotor systems.

Acknowledgments. We thank Przemysław Majewski for discussions on early stages of this work.

\section{References}

[1] Dyson, F. J., Lieb, E. H. and Simon, B.: J. Stat. Phys. 18, 335 (1978).

[2] Kennedy, T., Lieb, E. H. and Shastry, S.: J. Stat. Phys. 53, 1019 (1988).

[3] K. Osterwalder and R. Schrader: Comm. Math. Phys. 31, 83 (1974).

[4] Fröhlich, J., Simon, B. and Spencer, T.: Comm. Math. Phys. 50, 79 (1976).

[5] Pastur, L. A. and Khoruzhenko, B. A.: Theor. Math. Phys. 73, 1094 (1987).

[6] Neves, E. J. and Perez, J. F.: Phys. Lett. 114 A, 331 (1986).

[7] Kubo, K.: Phys. Rev. Lett. 61, 110 (1988). 
[8] Kennedy, T., Lieb, E. H. and Shastry, S.: Phys. Rev. Lett. 61, 2582 (1988).

[9] Schupp, P.: Quantum Systems Exactly. arXiv:math-ph/0206021.

[10] Wojtkiewicz, J.: Physica A 391, 5918 (2012).

[11] Wojtkiewicz, J.: Eur. Phys. J. B 44, 501 (2005).

[12] Sachdev, S.: Quantum Phase Transitions. Cambridge University Press, 1999.

[13] Mermin, N. D. and Wagner, H.: Phys. Rev. Lett. 17, 1133 (1966).

[14] Reed, M. and Simon, B.: Methods of modern Mathematical Physics, I: Functional analysis. Academic Press, New York - London, 1972.

[15] Reed, M. and Simon, B.: Methods of modern Mathematical Physics, II: Fourier analysis, selfadjointness. Academic Press, New York - London, 1975.

[16] Reed, M. and Simon, B.: Methods of modern Mathematical Physics, IV: Analysis of operators. Academic Press, New York - London, 1975.

[17] Schatten, R.: Norm Ideals of Completely Continuous Operators. Springer-Verlag, Berlin - New York, 1960.

[18] Dunford, N. and Schwartz, J. T.: Linear Operators, Part II: Spectral Theory. Interscience 1963.

[19] Kato, T.: Perturbation Theory for Linear Operators. Springer-Verlag, Berlin Heidelberg New York 1995.

[20] Aizenman, M., Lieb, E.H., Seiringer, R., Solovej, J. P. and Yngvason, J.: Phys. Rev. A 70, 023612 (2004).

[21] Verbeure, A. and Zagrebnov, V.: J. Phys. A Math. Gen. 28, 5415-5421 (1995).

[22] Alet, F. and Sorensen, E. S.: Phys. Rev. E 67, 015701(R) (2003)

[23] Nicolaescu L., Lectures on the Geometry of Manifolds, World Scientific 2007.

[24] Kosterlitz, J. M. and Thouless, D. J.: J. Phys. C 6, 1181-1203 (1973).

[25] Fröhlich, J. and Spencer, T.: Comm. Math. Phys. 81, 527-602 (1981). 